\title{
Price Discounts in Exchange for Reduced Customer Demand Variability and Applications to Advance Demand Information Acquisition
}

\author{
Sumit Kunnumkal \\ School of Operations Research and Industrial Engineering, \\ Cornell University, Ithaca, NY 14853, USA \\ Huseyin Topaloglu \\ School of Operations Research and Industrial Engineering, \\ Cornell University, Ithaca, NY 14853, USA \\ Email: ht88@cornell.edu, Tel: 607-255-0698, Fax: 607-255-9129
}

January 4, 2007

\begin{abstract}
We consider a supplier and a customer operating under a service agreement that requires the supplier to cover the random customer demand with high probability. To fulfill the service agreement, the supplier carries a certain amount of safety stock. The customer has some bearing on its demand variability, possibly through activities such as acquiring advance demand information, employing more sophisticated forecasting techniques or smoothing its product consumption, but these activities bring an extra cost to the customer. Since a reduction in the customer demand variability helps the supplier reduce its safety stock, the supplier is willing to offer a price discount in exchange for reduced demand variability. We examine a pricing scheme where the supplier assesses its potential cost savings from a reduction in the customer demand variability and returns a fraction of these cost savings back to the customer through a price discount. We show that both parties realize cost savings under such a pricing scheme, examine the efficiency issues and consider the case where the customer does not reveal certain cost components accurately.
\end{abstract}


Although conventional inventory models treat the customer demand as an exogenous random process, there are many situations where the customer can affect certain aspects of its demand process through activities such as acquiring advance demand information, employing more sophisticated forecasting techniques or smoothing its product consumption. Essentially, the outcome of these activities is to reduce the customer demand variability, which, in turn, benefits the customer through increased fill rates and the supplier through reduced inventories. However, although both the supplier and the customer benefit from these activities, the costs associated with them are usually born only by the customer. Consequently, the customer may pursue these activities in a much more limited scope than the supplier desires, and the supplier may have to share the costs of these activities with the customer or provide other incentives.

In this paper, we consider a supplier and a customer operating under a service agreement that requires the supplier to cover the random customer demand in each time period with high probability. The customer has some bearing on its demand variability, but this requires the customer to incur an extra cost. Early in the paper, we do not explicitly specify how the customer can affect its demand variability, but we later give a specific example where the customer can do this through advance demand information acquisition. A reduction in the customer demand variability helps the supplier reduce the safety stock that it needs to fulfill the service agreement, and the supplier is willing to offer a price discount to motivate the customer. We propose a pricing scheme that is motivated by the idea that the supplier should return a fraction of its potential cost savings that can be realized by the reduction in the demand variability back to the customer through a price discount. The result is a pricing scheme where the price charged to the customer is a linear function of the standard deviation of the customer demand. We show that both the supplier and the customer realize cost savings under such a pricing scheme. We examine the efficiency issues and consider the case where the customer does not reveal certain cost components accurately.

Our work is motivated by the relationship between a just-in-time manufacturer and its raw material supplier. In each time period, the supplier faces the demand for the raw material that the manufacturer needs for production in the current time period. Through better planning and forecasting techniques, the manufacturer can anticipate the need for the raw material in the future and reduce its demand variability, which, in turn, decreases the safety stock of the supplier. In our setting, the price discount is initiated by the supplier with the goal of reducing its safety stock requirements. Price discounts initiated by the suppliers often occur in industries where there are only a few suppliers and a few consumers of some raw material, and a specific supplier-consumer pair operates in a close relationship. Such situations are common in high-tech industries. As pointed by Lal and Staelin (1984), a large supplier may also initiate a price discount when supplying many 
small consumers. Our results may also be applicable in this setting by interpreting the customer demand in this paper as the total demand from all consumers.

The literature on exploiting different forms of information to increase the efficiency of inventory systems is quite rich. Several papers quantify the value of real-time demand information coming from the retailers in a supply chain. Lee et al. (2000) consider a two-stage supply chain where the customer demands at different time periods are correlated and show that the value of real-time demand information is significant especially when the demand quantities in different time periods are highly correlated. Cachon and Fisher (2000) study a distribution system with one warehouse and multiple retailers, and point out that the value of real-time demand information can be small when compared with the benefits of reducing the lead times or batch sizes. Gavirneni et al. (1999) quantify the value of real-time demand information in capacitated production and distribution systems. Bourland et al. (1999) consider a setting where the ordering cycles of the supplier and the retailer are not aligned. They show that the supplier can make better ordering decisions if it knows the amount of demand that occurs at the retailer between the time of the last order of the retailer and the time that the supplier places an order. Another form of information that is used to increase the efficiency of inventory systems is the forecast updates. Gullu (1996) and Gullu (1997) study production and distribution systems where the demand forecasts get more accurate over time and show that the inventory costs can be reduced if one explicitly takes the forecast updates into consideration.

There is also work on quantifying the value of advance demand information. Hariharan and Zipkin (1995) consider a continuous-time inventory control model where the customer demand becomes known $\tau$ time periods in advance. They refer to $\tau$ as the "demand lead time" and show that the effect of increasing the demand lead time is exactly the same as the effect of decreasing the supply lead time. Karaesmen et al. (2002) and Karaesmen et al. (2004) study production systems with advance demand information where the main difference from Hariharan and Zipkin (1995) is the limited production capacity modeled through a single-server queue. Ozer (2003) studies the value of advance demand information in distribution systems. Gilbert and Ballou (1999) examine the reductions in the inventory costs due to advance customer commitment and give a queueing model to assess the impact of advance customer commitment on the capacity requirements. Van Donselaar et al. (2001) and Thonemann (2002) consider the value of advance information in a project environment. Several companies submit proposals for projects. If a company manages to get a project, then it needs raw materials from its suppliers. The companies are able to share information about their proposals with their suppliers, although it is not certain that they will get the project. These papers quantify the value of such imperfect advance demand information to the suppliers.

Smoothing the orders is another method to increase the efficiency of inventory systems. Balakr- 
ishnan et al. (2004) and Disney et al. (2006) consider models where the retailer computes its order quantity according to a base-stock policy, but its actual order quantity is a convex combination of the order quantities computed in a certain number of past time periods. The goal is to reduce the variability of the orders that the supplier receives. Graves et al. (1998) describe a similar idea in a materials requirement planning environment with limited capacity. Boute et al. (2007) investigate the benefit from smoothing the orders in a make-to-order environment where the production lead time for the supplier depends on the orders placed by the retailer. An interesting point raised by this paper, and also by Bertrand (1986), is that although smoothing the orders is beneficial to the supplier, it may not be beneficial to the retailer since it restricts the ordering pattern of the retailer. Closely related to this body of research is the work on using sophisticated forecasting methods to deal with the bullwhip effect by reducing the variability of the orders that the supplier receives. Zhang (2004) characterizes the effect of different forecasting methods on the magnitude of the bullwhip effect. Dejonckheere et al. (2003) show that the bullwhip effect is inevitable as long as one uses a base-stock policy and propose alternative policies.

Lastly, the literature on setting up optimal price discounts is related to our work. Monahan (1984) studies a setting where a customer places orders to a supplier according to the economic order quantity and shows that the supplier can realize cost savings by offering price discounts to persuade the customer to place larger orders. Monahan (1984) assumes that the supplier and the customer have the same ordering cycles, but Lee and Rosenblatt (1986) generalize this model to allow the customer to order more frequently than the supplier. These two papers focus on maximizing the cost savings of the supplier. The supplier sets up the price discount scheme so that the customer, after the price discount, is only not worse off than it was before the price discount. Lal and Staelin (1984) and Dada and Srikanth (1987), on the other hand, consider the total cost of the supplier and the customer. They show how to set up a price discount scheme under which the ordering decisions of the supplier and the customer jointly minimize the total system cost. Weng (1995) extends this work by assuming that the demand is sensitive to price. An important point is that the papers mentioned in this paragraph do not provide a natural mechanism through which the cost savings from the price discount are shared between the supplier and the customer. Chen (2001) and Cheung (1998) present models that are useful when deciding how much price discount should be given to the customers who are willing to wait for the fulfillment of their orders. Klastorin et al. (2002) consider a case where the supplier is willing to offer a price discount if the customer plans the timing of its orders to coincide with the timing of the inventory replenishments of the supplier.

In this paper, we make the following research contributions. 1) We analyze a model that captures the relationship between a supplier and a customer where the supplier is willing to reduce the price if 
the customer reduces its demand variability. We propose a pricing scheme under which both parties realize cost savings. 2) We show that the cost savings of the supplier are larger than those of the customer under the proposed pricing scheme. In this pricing scheme, the supplier decides how the price will be adjusted according to the customer demand variability. Consequently, the supplier can be viewed as the dominant party and this dominance is reflected in its cost savings. 3) We show that if the supplier and the customer repeatedly use the proposed pricing scheme, then the customer eventually keeps the standard deviation of its demand at the level that minimizes the total system cost. 4) Realizing that setting up the proposed pricing scheme requires the supplier to know all cost parameters of the customer, we consider the case where the customer does not reveal a crucial cost component accurately. We show that both parties continue to realize cost savings under certain assumptions, and if the two parties repeatedly use the proposed pricing scheme, then the motivation for the customer to distort the cost component diminishes.

In Section 1, we formulate the model and derive the cost functions of the supplier and the customer. Section 2 describes the pricing scheme and shows that both parties realize cost savings under this pricing scheme. Section 3 considers the case where the customer does not reveal a crucial cost component accurately and presents results similar to those in Section 2. Section 4 shows the application of our model to a case where the customer can reduce its demand variability by acquiring advance demand information. In Section 5, we present numerical examples.

\section{Model Formulation}

We consider the relationship between a supplier and a customer operating under a service agreement that requires the supplier to cover the random customer demand in each time period with high probability. The customer has some bearing on its demand variability, but this requires the customer to incur an extra cost. This section does not explicitly specify how the customer can affect its demand variability, but Section 4 gives a specific example in the advance demand information acquisition context. To motivate the customer to reduce its demand variability, the supplier is willing to offer a price discount. We are interested in setting up a pricing scheme that results in cost savings for both parties. Sections 1 and 2 assume that all cost components are common information, or equivalently, the supplier and the customer are required by law or contracts to reveal their cost components accurately. Section 3 considers the case where the customer falsely reveals a crucial cost component.

We let $D_{t}$ be the customer demand in time period $t$ and assume that $D_{t}$ is normally distributed with mean $\mu$ and standard deviation $x$, where $x$ is a decision variable for the customer. To keep the standard deviation of its demand at $x$, the customer incurs a cost of $\mu \mathcal{C}(x)$ per time period. Therefore, one can view $\mu \mathcal{C}(x)$ as the cost of recurring operational activities or the periodic payment 
for an initial investment of the customer to keep the standard deviation of its demand at $x$. The customer chooses a value for $x$ once and does not change this value. To simplify the discussion, we assume that $x$ is constrained to lie in the interval $[0, b]$ for some finite $b$. As will be clear shortly, the multiplier $\mu$ in $\mu \mathcal{C}(\cdot)$ is simply for normalization.

We denote the first, second and third derivatives of the function $f(\cdot)$ by $\dot{f}(\cdot), \ddot{f}(\cdot)$ and $\dddot{f}(\cdot)$, respectively. When we say that $f(\cdot)$ is differentiable over the interval $[l, u]$, we mean that $f(\cdot)$ is differentiable over the interval $(l, u)$, right-differentiable at $l$ and left-differentiable at $u$. We assume that $\mathcal{C}(\cdot)$ is differentiable three times over $[0, b]$, and $0 \leq \mathcal{C}(x)<\infty,-\infty<\dot{\mathcal{C}}(x)<0,0<\ddot{\mathcal{C}}(x)<\infty$, $-\infty<\dddot{\mathcal{C}}(x)<0$ and $[\ddot{\mathcal{C}}(x)]^{2}<\dot{\mathcal{C}}(x) \dddot{\mathcal{C}}(x) \leq 2[\ddot{\mathcal{C}}(x)]^{2}$ for all $x \in[0, b]$. The assumptions on the signs of $\mathcal{C}(\cdot), \dot{\mathcal{C}}(\cdot)$ and $\ddot{\mathcal{C}}(\cdot)$ are intuitive. We need the other assumptions to ensure that certain optimization problems we encounter are convex. These assumptions are somewhat limiting, but they are satisfied by a variety of functions. For example, $\mathcal{C}(x)=\rho[x+\eta]^{-\alpha}$ and $\mathcal{C}(x)=\rho \mathrm{e}^{-\alpha x}-\gamma x$ with strictly positive $\rho, \eta, \alpha$ and $\gamma$, and small enough $\gamma$ satisfy these assumptions. It may also be possible to approximate a variety of functions by using a function that satisfies our assumptions. For example, although a linear function does not satisfy our assumptions, we can use $\rho[x+\eta]^{-\alpha}$ for judicious choices of $\rho, \eta$ and $\alpha$ to approximate a linear function over the interval $[0, b]$.

The service agreement requires the supplier to cover the customer demand in each time period with probability $\beta$. The unsatisfied customer demand is backlogged. Due to disruptions in its operations, the customer incurs a cost of $\pi$ per unit backlogged per time period. The supplier replenishes its inventory from an infinite source through a lead time of $\ell$ time periods. The unit cost of the replenishments is $c$. The holding cost of the supplier is $v$ per unit per time period. The unit price charged to the customer is $p$. We do not discount the future costs, and concentrate on the expected costs incurred by the supplier and the customer per time period. This assumption is appropriate when the discount factor is close to one.

The following events occur in time period $t$. First, the supplier receives the replenishment that it ordered in time period $t-\ell$. Second, the customer demand occurs and the supplier meets the demand as much as possible from its on-hand inventory. The excess demand is backlogged. Third, the supplier places a replenishment order. The holding and backlogging costs are calculated over the ending inventory. To characterize the inventory policy of the supplier, we define the following.

$O_{t}, B_{t}=$ On-hand inventory and backlogs of the supplier after covering the demand in time period $t$.

$T_{t}=$ Total in-transit replenishments of the supplier after receiving the replenishment and before placing a replenishment order in time period $t$. 
$L_{t}, P_{t}=$ Inventory level and inventory position of the supplier after covering the demand and before placing a replenishment order in time period $t$. That is, $L_{t}=O_{t}-B_{t}$ and $P_{t}=O_{t}-B_{t}+T_{t}$.

In time period $t$, the policy of the supplier is to order $S_{t}-P_{t}$ units, where $S_{t}$ is the base-stock level of the supplier. We shortly show that we have $S_{t}-P_{t} \geq 0$ and the order quantity is positive. Base-stock policies are optimal in many situations (Zipkin (2000)), but we are primarily motivated by the fact that a base-stock policy provides a simple way for the supplier to maintain the service level $\beta$. A standard computation shows that

$$
L_{t+\ell}=O_{t}-B_{t}+\left[S_{t}-P_{t}\right]+T_{t}-\sum_{s=t+1}^{t+\ell} D_{s}=S_{t}-\sum_{s=t+1}^{t+\ell} D_{s} .
$$

To maintain the service level $\beta$ in time period $t+\ell$, the supplier chooses $S_{t}$ such that $\mathbb{P}\left\{L_{t+\ell} \geq 0\right\}=$ $\mathbb{P}\left\{S_{t}-\sum_{s=t+1}^{t+\ell} D_{s} \geq 0\right\}=\beta$, where we use $\mathbb{P}\{\cdot\}$ to denote the probability of an event. Assuming that the demands in different time periods are independent, this implies that $S_{t}=\ell \mu+z \sqrt{\ell} x$, where $z$ is the $\beta$-th quantile of the standard normal distribution.

Since the replenishment quantity of the supplier in time period $t-1$ is $S_{t-1}-P_{t-1}$, we have $P_{t}=P_{t-1}+\left[S_{t-1}-P_{t-1}\right]-D_{t}=S_{t-1}-D_{t}$. Therefore, the replenishment quantity of the supplier in time period $t$ is $S_{t}-P_{t}=S_{t}-\left[S_{t-1}-D_{t}\right]=D_{t}$, which is positive as long as the demand is positive. Denoting the expectation of a random variable by $\mathbb{E}\{\cdot\}$ and $\max \{0, x\}$ by $[x]^{+}$, and letting $N$ be the standard normal random variable, the expected cost incurred by the supplier in time period $t$ is

$$
\begin{aligned}
c \mathbb{E}\left\{S_{t}-\left[S_{t-1}-D_{t}\right]\right\}+v \mathbb{E}\left\{\left[L_{t}\right]^{+}\right\}-p \mathbb{E}\left\{D_{t}\right\} & =c \mu+v \mathbb{E}\left\{\left[\ell \mu+z \sqrt{\ell} x-\sum_{s=t-\ell+1}^{t} D_{s}\right]^{+}\right\}-p \mu \\
& =c \mu+v \sqrt{\ell} \mathbb{E}\left\{[z-N]^{+}\right\} x-p \mu \\
& =c \mu+g \mu x-p \mu
\end{aligned}
$$

where the first equality uses (1), the second equality uses the fact that $\sum_{s=t-\ell+1}^{t} D_{s}$ is normally distributed with mean $\ell \mu$ and standard deviation $\sqrt{\ell} x$, and we let $g=v \sqrt{\ell} \mathbb{E}\left\{[z-N]^{+}\right\} / \mu$ in the third equality. Since the future costs are not discounted, the expressions above assume that the revenue from the backlogged demand is collected as soon as the backlog occurs. On the other hand, the expected cost incurred by the customer in time period $t$ is

$$
\begin{aligned}
\mu \mathcal{C}(x)+\pi \mathbb{E}\left\{\left[-L_{t}\right]^{+}\right\}+p \mathbb{E}\left\{D_{t}\right\} & =\mu \mathcal{C}(x)+\pi \mathbb{E}\left\{\left[\sum_{s=t-\ell+1}^{t} D_{s}-\ell \mu-z \sqrt{\ell} x\right]^{+}\right\}+p \mu \\
& =\mu \mathcal{C}(x)+\pi \sqrt{\ell} \mathbb{E}\left\{[N-z]^{+}\right\} x+p \mu \\
& =\mu \mathcal{C}(x)+h \mu x+p \mu,
\end{aligned}
$$

where we let $h=\pi \sqrt{\ell} \mathbb{E}\left\{[N-z]^{+}\right\} / \mu$ in the third equality. The customer does not incur any holding cost because it uses the product as soon as it is received from the supplier. 
The supplier and the customer concentrate on minimizing the cost functions in (2) and (3), respectively. Dropping the constant $c \mu$ in (2), and normalizing (2) and (3) by dividing by $\mu$ for brevity, we henceforth denote the cost functions of the supplier and the customer by $g x-p$ and $\mathcal{C}(x)+h x+p$, respectively. Our inventory model is clearly somewhat stylized, but it captures the essential relationship between the demand variability and the costs of the supplier and the customer.

\section{Pricing Scheme}

Let $\bar{x}$ be the current standard deviation of the customer demand and $\bar{p}$ be the current price that the supplier charges to the customer. If the customer is willing to reduce the standard deviation of its demand to $x$, then the cost incurred by the supplier decreases by $g \bar{x}-g x$. We propose a pricing scheme where the supplier transfers a fixed fraction of these cost savings to the customer by reducing the price to $\bar{p}-\widetilde{r}[g \bar{x}-g x]$, where the discount parameter $\widetilde{r}$ is a decision variable for the supplier. For brevity, we let $r=\widetilde{r} g$ and use $\bar{p}-r \bar{x}+r x$ to denote the pricing scheme adopted by the supplier.

Therefore, the supplier, knowing all cost components of itself and the customer, first chooses a discount parameter $r$ and reveals the pricing scheme $\bar{p}-r \bar{x}+r x$ to the customer, which is to say that if the customer sets the standard deviation of its demand to $x$, then the supplier charges the price $\bar{p}-r \bar{x}+r x$. Then, the customer, knowing how the supplier adjusts the price, decides to what level the standard deviation of its demand should be set. In this section, we show that both the supplier and the customer realize cost savings under such a pricing scheme, and the supplier, by having the power to dictate the set of standard deviation-price pairs that can be chosen by the customer, realizes larger cost savings than does customer. We also examine whether the standard deviation that minimizes the total system cost can be generated through the proposed pricing scheme. It is important to note that the discount parameter $r$ characterizes how the cost savings from the proposed pricing scheme are allocated between the supplier and the customer. The fact that $r$ is a decision variable for the supplier is different from some of the work in the supply chain contracts literature. Cachon (1998) and Tsay et al. (1998) give nice overviews of this literature.

To avoid trivialities, we assume that $\dot{\mathcal{C}}(0)+g+h<0<\dot{\mathcal{C}}(b)+g+h$, which means that if the standard deviation of the customer demand is $b$ (or 0 ), then the total marginal benefit of the supplier and the customer from reducing the standard deviation of the customer demand is greater (or less) than 0 . In this case, noting that the total system cost function is $[g x-p]+[\mathcal{C}(x)+h x+p]$, the derivative of the total system cost function is strictly negative at $x=0$ and strictly positive at $x=b$. Therefore, $x^{s}$, the minimizer of the total system cost function, satisfies the first order condition

$$
\dot{\mathcal{C}}\left(x^{s}\right)+g+h=0
$$

and we have $0<x^{s}<b$. We note that we have $g+h>0$ because of the assumption that 
$\dot{\mathcal{C}}(b)+g+h>0$. We also assume that the current standard deviation of the customer demand is large in the sense that $x^{s}<\bar{x} \leq b$. Using the same arguments, one can show that all of our results continue to hold when $0 \leq \bar{x}<x^{s}$, but we do not analyze this case for brevity.

\subsection{Preliminaries}

If the supplier adopts the pricing scheme $\bar{p}-r \bar{x}+r x$, then the customer decides to what level the standard deviation of its demand should be set by solving

$$
\min _{x \in[0, b]} \mathcal{C}(x)+h x+\bar{p}-r \bar{x}+r x .
$$

Since $\ddot{\mathcal{C}}(\cdot)>0$, the optimal solution to this problem is unique and we use $X(r)$ to denote the optimal solution as a function of the discount parameter $r$. Since the customer sets the standard deviation of its demand to $X(r)$ in response to the pricing scheme $\bar{p}-r \bar{x}+r x$, the supplier chooses the discount parameter by solving

$$
\min _{r} g X(r)-[\bar{p}-r \bar{x}+r X(r)] .
$$

We begin by characterizing the basic properties of problems (5) and (6).

Lemma 1 1) If $\dot{\mathcal{C}}(0)+r+h \leq 0 \leq \dot{\mathcal{C}}(b)+r+h$, then $X(r)$ uniquely satisfies

$$
\dot{\mathcal{C}}(X(r))+r+h=0 .
$$

Therefore, $X(\cdot)$ is differentiable over the interval $[-\dot{\mathcal{C}}(b)-h,-\dot{\mathcal{C}}(0)-h]$.

2) The objective function of problem (6) is differentiable and strictly convex over the interval $[-\dot{\mathcal{C}}(b)-$ $h,-\dot{\mathcal{C}}(0)-h]$.

3) The optimal solution to problem (6) lies in the interval $(-\dot{\mathcal{C}}(b)-h,-\dot{\mathcal{C}}(0)-h)$.

Proof We defer the proof of the third part to the appendix. If $\dot{\mathcal{C}}(0)+r+h \leq 0 \leq \dot{\mathcal{C}}(b)+r+h$, then the derivative of the objective function of problem (5) is negative at $x=0$ and positive at $x=b$. Therefore, $X(r)$ satisfies the first order condition in (7). Differentiability of $X(\cdot)$ follows from (7) and this establishes the first part. Since $X(\cdot)$ is differentiable over the interval $[-\dot{\mathcal{C}}(b)-h,-\dot{\mathcal{C}}(0)-h]$, the objective function of problem (6) is differentiable over this interval as well. Dropping the constant term, we write the objective function of problem (6) as $[g+h] X(r)+r \bar{x}-[r+h] X(r)$. We now show that $\ddot{X}(\cdot)>0$ and $[r+h] X(r)$ is a concave function of $r$, which establishes the second part. Differentiating (7) with respect to $r$ two times, we obtain

$$
\begin{array}{r}
\dot{X}(r) \ddot{\mathcal{C}}(X(r))+1=0 \\
\ddot{X}(r) \ddot{\mathcal{C}}(X(r))+[\dot{X}(r)]^{2} \dddot{\mathcal{C}}(X(r))=0 .
\end{array}
$$


Therefore, since $\ddot{\mathcal{C}}(\cdot)>0$ and $\dddot{\mathcal{C}}(\cdot)<0$, we have $\dot{X}(\cdot)<0$ and $\ddot{X}(\cdot)>0$. Differentiating $[r+h] X(r)$ with respect to $r$ two times, we obtain

$$
2 \dot{X}(r)+[r+h] \ddot{X}(r)=\dot{X}(r)\left\{2-\frac{[r+h] \dot{X}(r) \dddot{\mathcal{C}}(X(r))}{\ddot{\mathcal{C}}(X(r))}\right\}=\dot{X}(r)\left\{2-\frac{\dot{\mathcal{C}}(X(r)) \dddot{\mathcal{C}}(X(r))}{[\ddot{\mathcal{C}}(X(r))]^{2}}\right\} \leq 0
$$

where the first equality uses (9), and the second equality uses (7) and (8). The final inequality follows from the assumption that $\dot{\mathcal{C}}(x) \dddot{\mathcal{C}}(x) \leq 2[\ddot{\mathcal{C}}(x)]^{2}$.

The optimal solution to problem (6) lies in the interval $(-\dot{\mathcal{C}}(b)-h,-\dot{\mathcal{C}}(0)-h)$, over which the objective function is differentiable. Therefore, we can use the first order condition to characterize the optimal solution to problem (6). The following lemma shows important properties of the optimal solutions to problems (5) and (6). We defer its proof to the appendix.

Lemma 2 Let $r_{o}$ be the optimal solution to problem (6) and $x_{o}=X\left(r_{o}\right)$.

1) We have $-\dot{\mathcal{C}}(\bar{x})-h<r_{o}<g$ and $x^{s}<x_{o}<\bar{x}$.

2) There exists an $\widetilde{x}$ satisfying $\mathcal{C}(\widetilde{x})+h \widetilde{x}+\bar{p}-r_{o} \bar{x}+r_{o} \widetilde{x}<\mathcal{C}(\bar{x})+h \bar{x}+\bar{p}$.

Lemma 2 implies that if the supplier adopts the pricing scheme $\bar{p}-r_{o} \bar{x}+r_{o} x$, then the customer responds to this pricing scheme by reducing the standard deviation of its demand from $\bar{x}$ to $x_{o}$. In this case, the cost incurred by the supplier decreases by $g \bar{x}-g x_{o}$ and the supplier can reduce the price by as much as $g \bar{x}-g x_{o}$. Since $r_{o}<g$ and $x_{o}<\bar{x}$, we have $r_{o} \bar{x}-r_{o} x_{o}<g \bar{x}-g x_{o}$ and the price reduction is indeed less than $g \bar{x}-g x_{o}$.

\subsection{Analysis of the pricing scheme}

We are now ready to show that if the customer behaves rationally, then both the supplier and the customer can realize cost savings by using the proposed pricing scheme.

Proposition 3 Let $r_{o}$ be the optimal solution to problem (6) and $x_{o}=X\left(r_{o}\right)$. Assume that the supplier adopts the pricing scheme $\bar{p}-r_{o} \bar{x}+r_{o} x$.

1) The costs of the supplier and the customer under the standard deviation-price pair $\left(x_{o}, \bar{p}-r_{o} \bar{x}+\right.$ $\left.r_{o} x_{o}\right)$ are strictly less than their costs under the standard deviation-price pair $(\bar{x}, \bar{p})$.

2) Assume that the customer sets the standard deviation of its demand to $\widetilde{x}$, which satisfies $\mathcal{C}(\widetilde{x})+$ $h \widetilde{x}+\bar{p}-r_{o} \bar{x}+r_{o} \widetilde{x}<\mathcal{C}(\bar{x})+h \bar{x}+\bar{p}$. (The existence of such an $\widetilde{x}$ is guaranteed by Lemma 2.) Then, the costs of the supplier and the customer under the standard deviation-price pair $\left(\widetilde{x}, \bar{p}-r_{o} \bar{x}+r_{o} \widetilde{x}\right)$ are strictly less than their costs under the standard deviation-price pair $(\bar{x}, \bar{p})$. 
Proof We first show the second part. Since $\ddot{\mathcal{C}}(\cdot)>0$ and $\mathcal{C}(\widetilde{x})+h \widetilde{x}+\bar{p}-r_{o} \bar{x}+r_{o} \widetilde{x}<\mathcal{C}(\bar{x})+h \bar{x}+\bar{p}$, we have $\dot{\mathcal{C}}(\bar{x})[\widetilde{x}-\bar{x}]<\mathcal{C}(\widetilde{x})-\mathcal{C}(\bar{x})<-\left[r_{o}+h\right][\widetilde{x}-\bar{x}]$. Having $\dot{\mathcal{C}}(\bar{x})[\widetilde{x}-\bar{x}]<-\left[r_{o}+h\right][\widetilde{x}-\bar{x}]$ implies that $\widetilde{x}-\bar{x}<0$, because if we have $\widetilde{x}-\bar{x}>0$, then we obtain $\dot{\mathcal{C}}(\bar{x})<-\left[r_{o}+h\right]$ and this contradicts Lemma 2. Since $r_{o}<g$ by Lemma 2 and $\widetilde{x}-\bar{x}<0$, we have $r_{o}[\widetilde{x}-\bar{x}]>g[\widetilde{x}-\bar{x}]$, which implies that $g \widetilde{x}-\left[\bar{p}-r_{o} \bar{x}+r_{o} \widetilde{x}\right]<g \bar{x}-\bar{p}$. Therefore, the supplier is strictly better off under the standard deviation-price pair $\left(\widetilde{x}, \bar{p}-r_{o} \bar{x}+r_{o} \widetilde{x}\right)$. The customer is strictly better off under the standard deviation-price pair $\left(\widetilde{x}, \bar{p}-r_{o} \bar{x}+r_{o} \widetilde{x}\right)$ by definition of $\widetilde{x}$. This establishes the second part.

Since $\ddot{\mathcal{C}}(\cdot)>0, x_{o}$ is the unique solution to the problem $\min _{x \in[0, b]} \mathcal{C}(x)+h x+\bar{p}-r_{o} \bar{x}+r_{o} x$. By Lemma 2, we have $x_{o} \neq \bar{x}$, which implies that $\mathcal{C}\left(x_{o}\right)+h x_{o}+\bar{p}-r_{o} \bar{x}+r_{o} x_{o}<\mathcal{C}(\bar{x})+h \bar{x}+\bar{p}$. Therefore, the first part follows from the second part by letting $\widetilde{x}=x_{o}$.

The second part of Proposition 3 is stronger than the first part. The first part shows that if the supplier chooses the discount parameter by solving problem (6) and the customer decides to what level the standard deviation of its demand should be set by solving problem (5), then both parties are better off. The second part shows that the customer does not need to set the standard deviation of its demand to the level that minimizes its cost. If the customer sets the standard deviation of its demand to any level that makes itself better off, then both parties are better off. In the next proposition, we compare the cost savings of the supplier with those of the customer.

Proposition 4 Let $r_{o}$ be the optimal solution to problem (6) and $x_{o}=X\left(r_{o}\right)$. We have

$$
\{\mathcal{C}(\bar{x})+h \bar{x}+\bar{p}\}-\left\{\mathcal{C}\left(x_{o}\right)+h x_{o}+\bar{p}-r_{o} \bar{x}+r_{o} x_{o}\right\}<\{g \bar{x}-\bar{p}\}-\left\{g x_{o}-\left[\bar{p}-r_{o} \bar{x}+r_{o} x_{o}\right]\right\} .
$$

Proof Since $\ddot{\mathcal{C}}(\cdot)>0$ and $\dddot{\mathcal{C}}(\cdot)<0$, we have $\mathcal{C}(\bar{x})+\dot{\mathcal{C}}(\bar{x})\left[x_{o}-\bar{x}\right]<\mathcal{C}\left(x_{o}\right)$ and $\dot{\mathcal{C}}\left(x_{o}\right)+\ddot{\mathcal{C}}\left(x_{o}\right)\left[\bar{x}-x_{o}\right]>$ $\dot{\mathcal{C}}(\bar{x})$. Noting that $x_{o}-\bar{x}<0$ by Lemma 2 , multiplying the second inequality by $x_{o}-\bar{x}$ and adding the two inequalities, we obtain $\mathcal{C}(\bar{x})-\mathcal{C}\left(x_{o}\right)-\dot{\mathcal{C}}\left(x_{o}\right)\left[\bar{x}-x_{o}\right]<\ddot{\mathcal{C}}\left(x_{o}\right)\left[\bar{x}-x_{o}\right]^{2}$.

Since $r_{o}$ is the optimal solution to problem (6), it satisfies the first order condition $g \dot{X}\left(r_{o}\right)+\bar{x}-$ $r_{o} \dot{X}\left(r_{o}\right)-X\left(r_{o}\right)=0$. Multiplying this equality by $\ddot{\mathcal{C}}\left(X\left(r_{o}\right)\right)$, we obtain

$$
\ddot{\mathcal{C}}\left(X\left(r_{o}\right)\right)\left[g \dot{X}\left(r_{o}\right)+\bar{x}-r_{o} \dot{X}\left(r_{o}\right)-X\left(r_{o}\right)\right]=-g+\ddot{\mathcal{C}}\left(X\left(r_{o}\right)\right)\left[\bar{x}-X\left(r_{o}\right)\right]-\dot{\mathcal{C}}\left(X\left(r_{o}\right)\right)-h=0,
$$

where we use (7) and (8). Therefore, we have

$$
\begin{aligned}
\{\mathcal{C}(\bar{x})+h \bar{x}+\bar{p}\}-\left\{\mathcal{C}\left(x_{o}\right)\right. & \left.+h x_{o}+\bar{p}-r_{o} \bar{x}+r_{o} x_{o}\right\} \\
& =\mathcal{C}(\bar{x})-\mathcal{C}\left(x_{o}\right)+h\left[\bar{x}-x_{o}\right]+\left[\dot{\mathcal{C}}\left(x_{o}\right)+h\right]\left[x_{o}-\bar{x}\right]<\ddot{\mathcal{C}}\left(x_{o}\right)\left[\bar{x}-x_{o}\right]^{2} \\
& =\left\{\ddot{\mathcal{C}}\left(x_{o}\right)\left[\bar{x}-x_{o}\right]-\dot{\mathcal{C}}\left(x_{o}\right)-h\right\}\left[\bar{x}-x_{o}\right]+\left[\dot{\mathcal{C}}\left(x_{o}\right)+h\right]\left[\bar{x}-x_{o}\right] \\
& =g\left[\bar{x}-x_{o}\right]-r_{o}\left[\bar{x}-x_{o}\right]=\{g \bar{x}-\bar{p}\}-\left\{g x_{o}-\left[\bar{p}-r_{o} \bar{x}+r_{o} x_{o}\right]\right\},
\end{aligned}
$$


where the first equality follows by (7), the inequality follows by the inequality we derive at the beginning of the proof and the third equality follows by (7) and (10).

In our setting, the supplier can be viewed as the dominant party in the sense that, by choosing the discount parameter, it dictates what standard deviation-price pairs can be chosen by the customer. Due this dominance, the cost savings of the supplier end up being larger than those of the customer. Nonetheless, we emphasize that since the customer is free to set the standard deviation of its demand to any level in $[0, b]$, it has the final word on the benefits of the pricing scheme. For example, the customer can set the standard deviation of its demand to $\bar{x}$, in which case the supplier charges price $\bar{p}$ and both parties are neither better nor worse off. Lal and Staelin (1984) give examples of industries where the supplier has the power to decide on the structure of the pricing scheme.

Another important question is whether $x^{s}$, the "socially optimal" standard deviation, can be achieved through our pricing scheme. The first part of Lemma 2 shows that if the supplier and the customer concentrate on minimizing their own cost functions, then our pricing scheme does not generate $x^{s}$. However, we now show that if the supplier and the customer use our pricing scheme repeatedly, then level to which the customer sets the standard deviation of its demand converges to $x^{s}$. Specifically, let the sequences $\left\{x_{n}\right\},\left\{p_{n}\right\}$ and $\left\{r_{n}\right\}$ be generated through

$$
x_{n+1}=X\left(r_{n}\right) \text { and } p_{n+1}=p_{n}-r_{n} x_{n}+r_{n} x_{n+1},
$$

where $r_{n}=\operatorname{argmin}_{r} g X(r)-\left[p_{n}-r x_{n}+r X(r)\right], x_{1}=\bar{x}$ and $p_{1}=\bar{p}$. This corresponds to the situation where the supplier and the customer start from the standard deviation-price pair $\left(x_{1}, p_{1}\right)$. The supplier adopts the pricing scheme $p_{1}-r_{1} x_{1}+r_{1} x$, in response to which the customer sets the standard deviation of its demand to $x_{2}$ and the supplier charges price $p_{2}$. When the time for next negotiation comes, the supplier adopts the pricing scheme $p_{2}-r_{2} x_{2}+r_{2} x$. The customer sets the standard deviation of its demand to $x_{3}$ and the supplier charges price $p_{3}$. We note that $X\left(r_{2}\right)$ still gives the response of the customer to the pricing scheme $p_{2}-r_{2} x_{2}+r_{2} x$, because

$$
X\left(r_{2}\right)=\underset{x \in[0, b]}{\operatorname{argmin}} \mathcal{C}(x)+h x+\bar{p}-r_{2} \bar{x}+r_{2} x=\underset{x \in[0, b]}{\operatorname{argmin}} \mathcal{C}(x)+h x+p_{2}-r_{2} x_{2}+r_{2} x .
$$

We have the following result.

Proposition 5 We have $\lim _{n \rightarrow \infty} x_{n}=x^{s}$.

Proof By Lemma 2, we have $x^{s}<x_{2}=X\left(r_{1}\right)<x_{1}$. Starting from the standard deviation-price pair $\left(x_{2}, p_{1}-r_{1} x_{1}+r_{1} x_{2}\right)$ and reapplying Lemma 2, we have $x^{s}<x_{3}=X\left(r_{2}\right)<x_{2}$. Continuing in this fashion, we obtain $x^{s}<\ldots<x_{n+1}<x_{n}<\ldots<x_{1}$. Therefore, $\left\{x_{n}\right\}$ converges to some 
$x^{*} \geq x^{s}$. We want to show that $x^{*}=x^{s}$. Assume that $x^{*}>x^{s}$. Since $\ddot{\mathcal{C}}(\cdot)>0$, (4) implies that $\dot{\mathcal{C}}\left(x^{*}\right)+g+h>\dot{\mathcal{C}}\left(x^{s}\right)+g+h=0$. Using the same argument used to derive (10), we can show that

$$
\dot{\mathcal{C}}\left(x_{n+1}\right)+g+h=\dot{\mathcal{C}}\left(X\left(r_{n}\right)\right)+g+h=\ddot{\mathcal{C}}\left(X\left(r_{n}\right)\right)\left[x_{n}-X\left(r_{n}\right)\right]=\ddot{\mathcal{C}}\left(x_{n+1}\right)\left[x_{n}-x_{n+1}\right]
$$

holds for all $n$. Since $\ddot{\mathcal{C}}(\cdot)>0, \dddot{\mathcal{C}}(\cdot)<0$ and $x^{*}<x_{n+1}<x_{n}$, we have $0<\dot{\mathcal{C}}\left(x^{*}\right)+g+h<\dot{\mathcal{C}}\left(x_{n+1}\right)+g+$ $h=\ddot{\mathcal{C}}\left(x_{n+1}\right)\left[x_{n}-x_{n+1}\right]<\ddot{\mathcal{C}}\left(x^{*}\right)\left[x_{n}-x_{n+1}\right]$. Therefore, we have $x_{n}-x_{n+1}>\left[\dot{\mathcal{C}}\left(x^{*}\right)+g+h\right] / \ddot{\mathcal{C}}\left(x^{*}\right)>0$ for all $n$, which contradicts the fact that $\left\{x_{n}\right\}$ converges.

Since $x^{s}$ is the minimizer of the total system cost function, if $x_{n}=x^{s}$ for some $n$, then neither the supplier nor the customer can be better off without making the other party worse off. Therefore, Proposition 5 shows that the supplier and the customer can "squeeze" the maximum possible savings from the system by using the proposed pricing scheme repeatedly. Furthermore, the proposed pricing scheme gives a natural mechanism to allocate the savings between the supplier and the customer.

\section{Manipulation of the Pricing Scheme}

In many settings, the cost component $\mathcal{C}(\cdot)$ is private information for the customer and the customer may not reveal this cost component accurately to the supplier. In this section, we show that distorting this cost component is beneficial for the customer. Furthermore, under certain assumptions, we show that both parties can still realize cost savings, even if the customer does not reveal the cost component $\mathcal{C}(\cdot)$ accurately. We also reexamine the ability of the proposed pricing scheme to generate $x^{s}$. The proofs in this section are similar to those in Section 2 and we defer most of them to the appendix.

\subsection{Preliminaries}

Assume that the customer reveals the cost component $\mathcal{C}(\cdot)$ to the supplier as $\lambda \mathcal{C}(\cdot)$, where $\lambda>0$ is a decision variable for the customer. Then, the belief of the supplier is that the customer solves

$$
\min _{x \in[0, b]} \lambda \mathcal{C}(x)+h x+\bar{p}-r \bar{x}+r x
$$

to decide to what level the standard deviation of its demand should be set. Since $\lambda>0$ and $\ddot{\mathcal{C}}(\cdot)>0$, the problem above has a unique optimal solution. The next lemma shows that this optimal solution can be captured by using the notation we previously defined.

Lemma 6 The optimal solution to problem (12) can be written as $X\left(\frac{r+h}{\lambda}-h\right)$.

Proof If $\dot{\mathcal{C}}(0)+\frac{r+h}{\lambda} \leq 0 \leq \dot{\mathcal{C}}(b)+\frac{r+h}{\lambda}$, then we have $\dot{\mathcal{C}}\left(X\left(\frac{r+h}{\lambda}-h\right)\right)+\frac{r+h}{\lambda}-h+h=0$ by (7), which implies that

$$
\lambda \dot{\mathcal{C}}\left(X\left(\frac{r+h}{\lambda}-h\right)\right)+r+h=0 .
$$


This shows that the first order condition to problem (12) is satisfied at $X\left(\frac{r+h}{\lambda}-h\right)$. Therefore, the optimal solution to problem (12) is $X\left(\frac{r+h}{\lambda}-h\right)$ when $\dot{\mathcal{C}}(0)+\frac{r+h}{\lambda} \leq 0 \leq \dot{\mathcal{C}}(b)+\frac{r+h}{\lambda}$. We consider the cases $\dot{\mathcal{C}}(0)+\frac{r+h}{\lambda}>0$ and $\dot{\mathcal{C}}(b)+\frac{r+h}{\lambda}<0$ in the appendix.

Since the supplier believes that the customer responds to the pricing scheme $\bar{p}-r \bar{x}+r x$ by setting the standard deviation of its demand to $X\left(\frac{r+h}{\lambda}-h\right)$, the supplier chooses the discount parameter by solving

$$
\min _{r} g X\left(\frac{r+h}{\lambda}-h\right)-\left[\bar{p}-r \bar{x}+r X\left(\frac{r+h}{\lambda}-h\right)\right] .
$$

Lemma 7 below shows that the optimal solution to problem (14) is unique and we use $R(\lambda)$ to denote the optimal solution to this problem as a function of the distortion parameter $\lambda$. Therefore, if the customer reveals the cost component $\mathcal{C}(\cdot)$ as $\lambda \mathcal{C}(\cdot)$, then the supplier adopts the pricing scheme $\bar{p}-R(\lambda) \bar{x}+R(\lambda) x$. We note that, contrary to what our notation suggests, the supplier does not need to know the value of $\lambda$ to solve problem (14). By (13), $X\left(\frac{r+h}{\lambda}-h\right)$ is the inverse of $\lambda \dot{\mathcal{C}}(\cdot)$ evaluated at $-[r+h]$. Therefore, the knowledge of $\lambda \mathcal{C}(\cdot)$, which implies the knowledge of $\lambda \dot{\mathcal{C}}(\cdot)$, is sufficient for the supplier to solve problem (14).

Finally, to decide how much to distort the cost component $\mathcal{C}(\cdot)$, the customer solves

$$
\min _{\lambda \in[\delta, \infty)} \mathcal{C}\left(X\left(\frac{R(\lambda)+h}{\lambda}-h\right)\right)+h X\left(\frac{R(\lambda)+h}{\lambda}-h\right)+\bar{p}-R(\lambda) \bar{x}+R(\lambda) X\left(\frac{R(\lambda)+h}{\lambda}-h\right),
$$

where $\delta$ is small and strictly positive. Lemma 8 below shows that it is enough to choose $\delta \leq 1$. The way that problem (15) is formulated implies that if the supplier chooses the discount parameter as $R(\lambda)$, then the customer sets the standard deviation of its demand to $X\left(\frac{R(\lambda)+h}{\lambda}-h\right)$, which is the minimizer of the cost function $\lambda \mathcal{C}(x)+h x+\bar{p}-R(\lambda) \bar{x}+R(\lambda) x$ (instead of setting the standard deviation of its demand to $X(R(\lambda))$, which is the minimizer of the cost function $\mathcal{C}(x)+h x+\bar{p}-$ $R(\lambda) \bar{x}+R(\lambda) x)$. The reason for this is that the supplier believes that the cost component $\mathcal{C}(\cdot)$ is $\lambda \mathcal{C}(\cdot)$, and the goal of the customer is to "appear" to be rational to the supplier by setting the standard deviation of its demand to $X\left(\frac{R(\lambda)+h}{\lambda}-h\right)$. This also makes it impossible for the supplier to infer the value of $\lambda$ by a "reverse" computation. We begin by characterizing the basic properties of problem (14).

Lemma 7 1) The objective function of problem (14) is differentiable and strictly convex over the interval $[-\lambda \dot{\mathcal{C}}(b)-h,-\lambda \dot{\mathcal{C}}(0)-h]$.

2) The optimal solution to problem (14) lies in the interval $(-\lambda \dot{\mathcal{C}}(b)-h,-\lambda \dot{\mathcal{C}}(0)-h)$.

Similar to Lemma 1, Lemma 7 enables us to concentrate on the objective function of problem (14) only over the interval $[-\lambda \dot{\mathcal{C}}(b)-h,-\lambda \dot{\mathcal{C}}(0)-h]$ and use the first order condition to characterize 
the optimal solution. The following lemma shows important properties of the optimal solutions to problems (12), (14) and (15).

Lemma 8 Let $\lambda_{o}$ be the optimal solution to problem $(15), r_{o}=R\left(\lambda_{o}\right)$ and $x_{o}=X\left(\frac{r_{o}+h}{\lambda_{o}}-h\right)$.

1) We have $1<\lambda_{o}<-[g+h] / \dot{\mathcal{C}}(\bar{x}),-\lambda_{o} \dot{\mathcal{C}}(\bar{x})-h<r_{o}<g$ and $x^{s}<x_{o}<\bar{x}$.

2) There exists an $\widetilde{x}$ satisfying $\lambda_{o} \mathcal{C}(\widetilde{x})+h \widetilde{x}+\bar{p}-r_{o} \bar{x}+r_{o} \widetilde{x}<\lambda_{o} \mathcal{C}(\bar{x})+h \bar{x}+\bar{p}$.

From this lemma, since $\lambda_{o}>1$, the customer prefers to overstate the cost component $\mathcal{C}(\cdot)$.

\subsection{Analysis of the pricing scheme under manipulation}

In the next result, we show that the supplier and the customer can still be better off by employing the proposed pricing scheme.

Proposition 9 Let $\lambda_{o}$ be the optimal solution to problem (15), $r_{o}=R\left(\lambda_{o}\right)$ and $x_{o}=X\left(\frac{r_{o}+h}{\lambda_{o}}-h\right)$. Assume that the supplier adopts the pricing scheme $\bar{p}-r_{o} \bar{x}+r_{o} x$.

1) The costs of the supplier and the customer under the standard deviation-price pair $\left(x_{o}, \bar{p}-r_{o} \bar{x}+\right.$ $\left.r_{o} x_{o}\right)$ are strictly less than their costs under the standard deviation-price pair $(\bar{x}, \bar{p})$.

2) Assume that the customer sets the standard deviation of its demand to $\widetilde{x}$, which satisfies $\lambda_{o} \mathcal{C}(\widetilde{x})+$ $h \widetilde{x}+\bar{p}-r_{o} \bar{x}+r_{o} \widetilde{x}<\lambda_{o} \mathcal{C}(\bar{x})+h \bar{x}+\bar{p}$. (The existence of such an $\widetilde{x}$ is guaranteed by Lemma 8.) Then, the costs of the supplier and the customer under the standard deviation-price pair $\left(\widetilde{x}, \bar{p}-r_{o} \bar{x}+r_{o} \widetilde{x}\right)$ are strictly less than their costs under the standard deviation-price pair $(\bar{x}, \bar{p})$.

Clearly, the customer increases its cost savings by distorting the cost component $\mathcal{C}(\cdot)$, otherwise we would have $\lambda_{o}=1$. However, when the customer does not reveal the cost component $\mathcal{C}(\cdot)$ accurately, the cost savings realized by the customer do not necessarily exceed the cost savings realized by the supplier. In fact, the relative magnitudes of the cost savings of the two parties can depend on the problem parameters and we do not have a result comparable to Proposition 4 when the customer does not reveal the cost component $\mathcal{C}(\cdot)$ accurately. Section 5 gives numerical examples illustrating the effects of the problem parameters on the cost savings of the two parties.

We close this section by examining the ability of the proposed pricing scheme to generate the socially optimal standard deviation. The first part of Lemma 8 shows that if the supplier and the customer concentrate on minimizing their own cost functions, then our pricing scheme does not generate $x^{s}$. However, if the supplier and the customer use our pricing scheme repeatedly, then the level to which the customer sets the standard deviation of its demand converges to $x^{s}$ and the 
motivation for the customer to distort the cost component $\mathcal{C}(\cdot)$ diminishes. Specifically, let the sequences $\left\{x_{n}\right\},\left\{p_{n}\right\}$ and $\left\{\lambda_{n}\right\}$ be generated through

$$
x_{n+1}=X\left(\frac{R_{n}\left(\lambda_{n}\right)+h}{\lambda_{n}}-h\right) \text { and } p_{n+1}=p_{n}-R_{n}\left(\lambda_{n}\right) x_{n}+R_{n}\left(\lambda_{n}\right) x_{n+1},
$$

where

$$
\begin{aligned}
R_{n}(\lambda)=\underset{r}{\operatorname{argmin}} g X\left(\frac{r+h}{\lambda}-h\right)-\left[p_{n}-r x_{n}+r X\left(\frac{r+h}{\lambda}-h\right)\right] \\
\lambda_{n}=\underset{\lambda \in[\delta, \infty)}{\operatorname{argmin}} \mathcal{C}\left(X\left(\frac{R_{n}(\lambda)+h}{\lambda}-h\right)\right)+h X\left(\frac{R_{n}(\lambda)+h}{\lambda}-h\right) \\
\quad+p_{n}-R_{n}(\lambda) x_{n}+R_{n}(\lambda) X\left(\frac{R_{n}(\lambda)+h}{\lambda}-h\right),
\end{aligned}
$$

$x_{1}=\bar{x}$ and $p_{1}=\bar{p}$. This corresponds to the situation where the supplier and the customer start from the standard deviation-price pair $\left(x_{1}, p_{1}\right)$. The customer reveals the cost component $\mathcal{C}(\cdot)$ as $\lambda_{1} \mathcal{C}(\cdot)$. The supplier adopts the pricing scheme $p_{1}-R_{1}\left(\lambda_{1}\right) x_{1}+R_{1}\left(\lambda_{1}\right) x$, in response to which the customer sets the standard deviation of its demand to $x_{2}$ and the supplier charges price $p_{2}$. When the time for next negotiation comes, the customer reveals the cost component $\mathcal{C}(\cdot)$ as $\lambda_{2} \mathcal{C}(\cdot)$. We have the following result.

Proposition 10 We have $\lim _{n \rightarrow \infty} x_{n}=x^{s}$ and $\lim _{n \rightarrow \infty} \lambda_{n}=1$.

\section{Applications to Advance Demand Information Acquisition}

This section shows how the results of Sections 2 and 3 apply to a situation where the supplier is willing to offer a price discount to the customer in exchange for advance demand information.

We assume that the customer demand in time period $t$ has the form

$$
D_{t}=\mu+\sqrt{y} A_{t}+\sqrt{1-y} Z_{t}
$$

where $y \in[0,1]$ is a decision variable for the customer, and $A_{t}$ and $Z_{t}$ are independent normally distributed random variables with mean zero and standard deviation $\sigma$. The value of the random variable $A_{t}$ becomes known in time period $t-\tau$, where $\tau$ is a positive time lag, but the value of the random variable $Z_{t}$ becomes known in time period $t$. In this way, $A_{t}$ captures the portion of the customer demand that is known in advance and $y$ captures the "amount" of advance demand information that the customer acquires. Therefore, $D_{t}$ has standard deviation $\sigma$ when "viewed" from a time period before $t-\tau$, but has standard deviation $\sigma \sqrt{1-y}$ when viewed from a time period after $t-\tau$ and before $t$. To avoid trivialities, we assume that $\tau \leq \ell$.

The specific form for $D_{t}$ in (19) may arise from the assumption that the customer demand in time period $t$ is the sum of the demands generated by $K$ independent "sources," and, in time period 
$t-\tau$, the customer acquires advance demand information for a fraction of these sources to find out whether they will generate demands in time period $t$. In this case, letting $q$ be the probability that a particular source generates a unit of demand and $y$ be the fraction of sources for which the customer acquires advance demand information, we have $D_{t}=\widetilde{A}_{t}+\widetilde{Z}_{t}$, where $\widetilde{A}_{t}$ and $\widetilde{Z}_{t}$ are independent binomially distributed random variables with parameters $(q, K y)$ and $(q, K[1-y])$, respectively. The values of the random variables $\widetilde{A}_{t}$ and $\widetilde{Z}_{t}$ become known in time periods $t-\tau$ and $t$, respectively. If $K$ is large, then we can approximate $\widetilde{A}_{t}$ and $\widetilde{Z}_{t}$ by normally distributed random variables with mean-standard deviation pairs $(q K y, \sqrt{q[1-q] K y})$ and $(q K[1-y], \sqrt{q[1-q] K[1-y]})$, respectively. Letting $\mu=q K$ and $\sigma=\sqrt{q[1-q] K}$, we obtain (19).

We assume that if the amount of advance demand information that the customer acquires is $y$, then the customer incurs a cost of $\mu \mathcal{C}(\sigma \sqrt{1-[\tau / \ell] y})$ per time period, where $\mathcal{C}(\cdot)$ satisfies the assumptions in Section 1. As will be clear shortly, the multipliers $\mu$ and $\sigma$ are simply for normalization. The multiplier $\sigma$ also ensures that the argument of $\mathcal{C}(\cdot)$ has standard deviation "units." We note that when the supplier places a replenishment order in time period $t$, the random portion of the customer demand over the lead time is $\sqrt{y} \sum_{s=t+\tau+1}^{t+\ell} A_{s}+\sqrt{1-y} \sum_{s=t+1}^{t+\ell} Z_{s}$, which has standard deviation $\sigma \sqrt{\ell-\tau y}$. Therefore, the argument of $\mathcal{C}(\cdot)$ is related to the standard deviation of the customer demand over the lead time. Although it limits the scope of our analysis, the specific form we choose for $\mathcal{C}(\cdot)$ is not too restrictive from the practical viewpoint. For example, $\mathcal{C}(\sigma \sqrt{1-[\tau / \ell] y})$ is increasing and convex in $y$ as long as $\mathcal{C}(\cdot)$ satisfies the assumptions in Section 1. Furthermore, it may be possible to approximate a variety of functions by using a function of the form $\mathcal{C}(\sigma \sqrt{1-[\tau / \ell] y})$ for a judicious choice of $\mathcal{C}(\cdot)$.

Using the same notation as in Section 1, the supplier chooses $S_{t}$ such that

$$
\begin{aligned}
\mathbb{P}\left\{L_{t+\ell} \geq 0 \mid A_{t+1}, \ldots, A_{t+\tau}\right\} & \\
& =\mathbb{P}\left\{S_{t}-\ell \mu-\sqrt{y} \sum_{s=t+1}^{t+\ell} A_{s}-\sqrt{1-y} \sum_{s=t+1}^{t+\ell} Z_{s} \geq 0 \mid A_{t+1}, \ldots, A_{t+\tau}\right\}=\beta .
\end{aligned}
$$

This implies that $S_{t}=\ell \mu+\sqrt{y} \sum_{s=t+1}^{t+\tau} A_{s}+z \sigma \sqrt{\ell-\tau y}$. Noting (1), we have

$$
L_{t}=S_{t-\ell}-\sum_{s=t-\ell+1}^{t} D_{s}=z \sigma \sqrt{\ell-\tau y}-\sqrt{y} \sum_{s=t-\ell+\tau+1}^{t} A_{s}-\sqrt{1-y} \sum_{s=t-\ell+1}^{t} Z_{s} .
$$

The expected cost incurred by the supplier in time period $t$ is

$$
\begin{aligned}
& c \mathbb{E}\left\{S_{t}-\left[S_{t-1}-D_{t}\right]\right\}+v \mathbb{E}\left\{\left[L_{t}\right]^{+}\right\}-p \mathbb{E}\left\{D_{t}\right\} \\
& =c \mathbb{E}\left\{\mu+\sqrt{y} A_{t+\tau}+\sqrt{1-y} Z_{t}\right\} \\
& \quad+v \mathbb{E}\left\{\left[z \sigma \sqrt{\ell-\tau y}-\sqrt{y} \sum_{s=t-\ell+\tau+1}^{t} A_{s}-\sqrt{1-y} \sum_{s=t-\ell+1}^{t} Z_{s}\right]^{+}\right\}-p \mu \\
& =c \mu+v \sqrt{\ell} \mathbb{E}\left\{[z-N]^{+}\right\} \sigma \sqrt{1-[\tau / \ell] y}-p \mu \\
& =c \mu+g \mu \sigma \sqrt{1-[\tau / \ell] y}-p \mu
\end{aligned}
$$


where the second equality uses the fact that $\sqrt{y} \sum_{s=t-\ell+\tau+1}^{t} A_{s}+\sqrt{1-y} \sum_{s=t-\ell+1}^{t} Z_{s}$ is normally distributed with mean zero and standard deviation $\sigma \sqrt{\ell-\tau y}$. Since we have $S_{t}-\left[S_{t-1}-D_{t}\right]=$ $\mu+\sqrt{y} A_{t+\tau}+\sqrt{1-y} Z_{t}$, the replenishment quantity in time period $t$ is normally distributed with mean $\mu$ and standard deviation $\sigma$, and it is positive with high probability as long as $\sigma$ is small compared to $\mu$. The expected cost incurred by the customer in time period $t$ is

$$
\begin{aligned}
\mu \mathcal{C} & (\sigma \sqrt{1-[\tau / \ell] y})+\pi \mathbb{E}\left\{\left[-L_{t}\right]^{+}\right\}+p \mathbb{E}\left\{D_{t}\right\} \\
& =\mu \mathcal{C}(\sigma \sqrt{1-[\tau / \ell] y})+\pi \mathbb{E}\left\{\left[\sqrt{y} \sum_{s=t-\ell+\tau+1}^{t} A_{s}+\sqrt{1-y} \sum_{s=t-\ell+1}^{t} Z_{s}-z \sigma \sqrt{\ell-\tau y}\right]^{+}\right\}+p \mu \\
& =\mu \mathcal{C}(\sigma \sqrt{1-[\tau / \ell] y})+\pi \sqrt{\ell} \mathbb{E}\left\{[N-z]^{+}\right\} \sigma \sqrt{1-[\tau / \ell] y}+p \mu \\
& =\mu \mathcal{C}(\sigma \sqrt{1-[\tau / \ell] y})+h \mu \sigma \sqrt{1-[\tau / \ell] y}+p \mu
\end{aligned}
$$

Letting $\sigma \sqrt{1-[\tau / \ell] y}=x,(20)$ and (21) have the same form as (2) and (3), and the results of Sections 2 and 3 apply. Comparing (2) and (3) with (20) and (21) shows that if the amount of advance demand information that the customer acquires is $y$, then the "effective" standard deviation of the customer demand is $\sigma \sqrt{1-[\tau / \ell] y}$. In the next section, we refer to $\sigma \sqrt{1-[\tau / \ell] y}$ simply as the standard deviation of the customer demand, although, in reality, the standard deviation of the customer demand is fixed at $\sigma$.

\section{$5 \quad$ Numerical Illustrations}

This section presents numerical examples to illustrate the behavior of the proposed pricing scheme. We use the setting in Section 4 with $\sigma=6, \ell=12, \tau=5, \beta=0.9, v=0.05, \pi=1.5, \mathcal{C}(x)=$ $\rho\left[x+10^{-6}\right]^{-4}$ and $\rho=8$. The small term $10^{-6}$ in $\left[x+10^{-6}\right]^{-4}$ ensures that $\mathcal{C}(\cdot)$ satisfies the assumptions in Section 1. We assume that the customer currently does not acquire advance demand information so that $\bar{x}=\sigma \sqrt{1-[\tau / \ell] 0}=6$. As a side remark, noting (20) and (21), a standard computation shows that the value of $z$ that minimizes the total system cost function

$$
\mu \mathcal{C}(\sigma \sqrt{1-[\tau / \ell] y})+\sqrt{\ell}\left[v \mathbb{E}\left\{[z-N]^{+}\right\}+\pi \mathbb{E}\left\{[N-z]^{+}\right\}\right] \sigma \sqrt{1-[\tau / \ell] y}+c \mu
$$

satisfies $\mathbb{P}\{z \geq N\}=\pi /[v+\pi]$ and the service agreement that minimizes the total system cost function requires the supplier to satisfy the customer demand with probability $1.5 /[0.05+1.5] \approx 0.97$. Therefore, the service agreement with $\beta=0.9$ that we consider in this section deviates somewhat from the service agreement that minimizes the total system cost function. We also note that although our results are on one set of parameters, they are representative of the behavior over a wide range of parameters. To give an idea about the magnitude of the cost savings, we normalize each cost figure by dividing by $[\mathcal{C}(\bar{x})+g \bar{x}+h \bar{x}] / 100$, which is the total system cost under the current standard deviation of the customer demand.

Letting $r_{o}$ be the optimal solution to problem (6) and $x_{o}=X\left(r_{o}\right)$, the cost savings of the supplier 

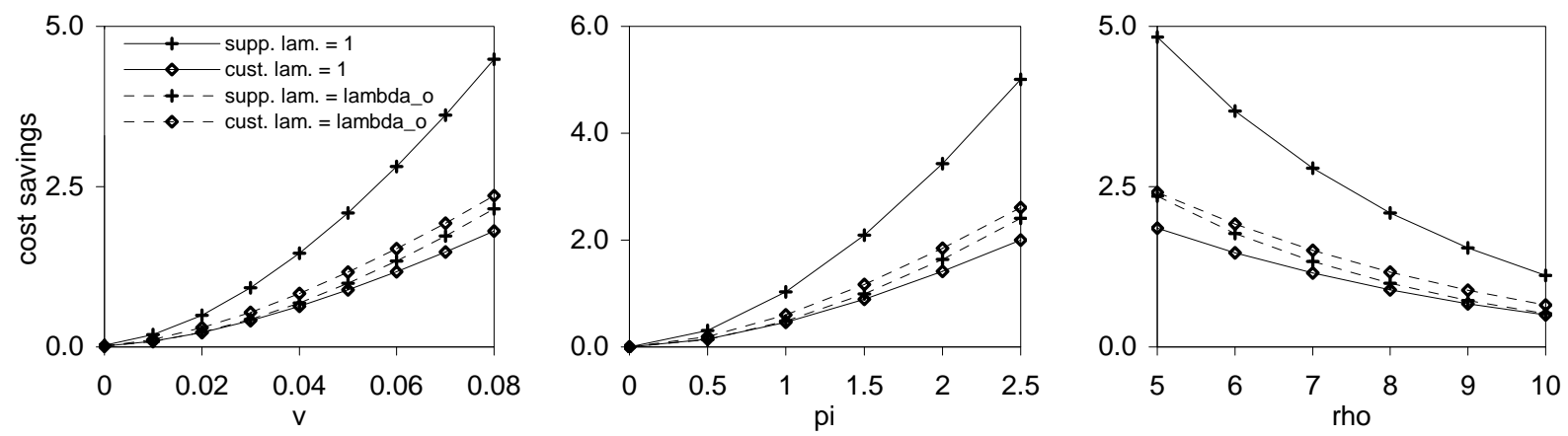

Figure 1: Cost savings realized by the supplier and the customer as a function of $v, \pi$ and $\rho$.

and the customer are $\{g \bar{x}-\bar{p}\}-\left\{g x_{o}-\left[\bar{p}-r_{o} \bar{x}+r_{o} x_{o}\right]\right\}$ and $\{\mathcal{C}(\bar{x})+h \bar{x}+\bar{p}\}-\left\{\mathcal{C}\left(x_{o}\right)+h x_{o}+\bar{p}-\right.$ $\left.r_{o} \bar{x}+r_{o} x_{o}\right\}$, respectively. In Figure 1, the solid data series show how these cost savings change with $v, \pi$ and $\rho$. We observe that both the supplier and the customer benefit from the proposed pricing scheme especially when the uncertainty in the customer demand costs highly either to the supplier $(v)$ or to the customer $(\pi)$, or when the cost of acquiring advance demand information $(\rho)$ is small.

Section 3 shows that it is beneficial for the customer not to reveal the cost component $\mathcal{C}(\cdot)$ accurately. If the customer reveals the cost component $\mathcal{C}(\cdot)$ as $\lambda \mathcal{C}(\cdot)$, then the supplier chooses the discount parameter as $R(\lambda)$, the customer sets the standard deviation of its demand to $X\left(\frac{R(\lambda)+h}{\lambda}-h\right)$, and the cost savings of the supplier and the customer become

$$
\begin{gathered}
\{g \bar{x}-\bar{p}\}-\left\{g X\left(\frac{R(\lambda)+h}{\lambda}-h\right)-\left[\bar{p}-R(\lambda) \bar{x}+R(\lambda) X\left(\frac{R(\lambda)+h}{\lambda}-h\right)\right]\right\} \text { and } \\
\{\mathcal{C}(\bar{x})+h \bar{x}+\bar{p}\}-\left\{\mathcal{C}\left(X\left(\frac{R(\lambda)+h}{\lambda}-h\right)\right)+h X\left(\frac{R(\lambda)+h}{\lambda}-h\right)+\bar{p}-R(\lambda) \bar{x}+R(\lambda) X\left(\frac{R(\lambda)+h}{\lambda}-h\right)\right\},
\end{gathered}
$$

respectively. In Figure 1, the solid data series show how these cost savings change with $v, \pi$ and $\rho$ when $\lambda=1$, whereas the dashed data series show how these cost savings change with $v, \pi$ and $\rho$ when $\lambda=\lambda_{o}$, where $\lambda_{o}$ is the optimal solution to problem (15). We observe that the customer increases its cost savings by revealing the cost component $\mathcal{C}(\cdot)$ as $\lambda_{o} \mathcal{C}(\cdot)$, but this results in a decrease in the cost savings of the supplier. As shown in Proposition 4, the cost savings of the supplier are always larger than those of the customer when $\lambda=1$, but this is not necessarily true when $\lambda=\lambda_{o}$.

Propositions 5 and 10 show that if the proposed pricing scheme is used repeatedly, then the level to which the customer sets the standard deviation of its demand converges to $x^{s}$. We let $\left\{x_{n}^{T}\right\}$ and $\left\{p_{n}^{T}\right\}$ be generated through (11), and $\left\{x_{n}^{F}\right\}$ and $\left\{p_{n}^{F}\right\}$ be generated through (16). Therefore, the standard deviation-price pairs $\left\{\left(x_{n}^{T}, p_{n}^{T}\right)\right\}$ and $\left\{\left(x_{n}^{F}, p_{n}^{F}\right)\right\}$ respectively correspond to the cases where the customer does and does not reveal the cost component $\mathcal{C}(\cdot)$ accurately. On the left side of Figure 2, we plot $\left\{x_{n}^{T}\right\}$ and $\left\{x_{n}^{F}\right\}$ as a function of $n$. Noting that the cumulative cost savings of the supplier and the customer over $n$ negotiations can respectively be written as $\left[g x_{1}^{i}-p_{1}^{i}\right]-\left[g x_{n}^{i}-p_{n}^{i}\right]$ and $\left[\mathcal{C}\left(x_{1}^{i}\right)+h x_{1}^{i}+p_{1}^{i}\right]-\left[\mathcal{C}\left(x_{n}^{i}\right)+h x_{n}^{i}+p_{n}^{i}\right]$ for $i \in\{T, F\}$, we plot these cumulative cost savings as 

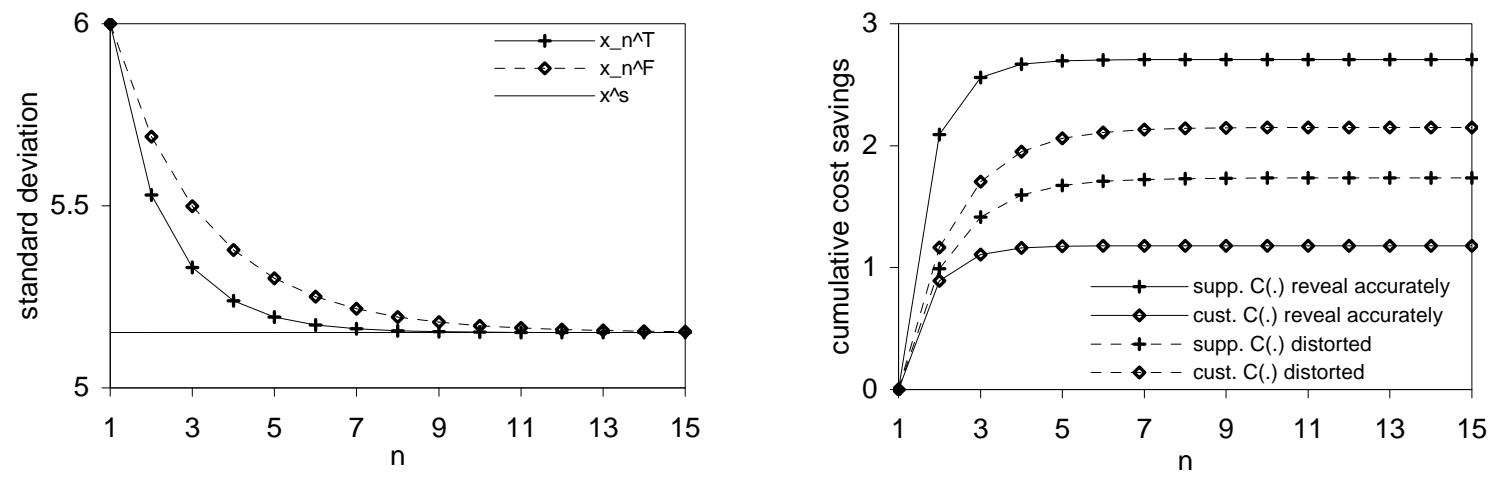

Figure 2: Standard deviation of the customer demand, and cost savings realized by the supplier and the customer when the proposed pricing scheme is used repeatedly.

a function of $n$ on the right side of Figure 2. Both $\left\{x_{n}^{T}\right\}$ and $\left\{x_{n}^{F}\right\}$ converge to $x^{s}$ before $n$ reaches 14-15. We observe that the customer can significantly increase its cost savings by not revealing the cost component $\mathcal{C}(\cdot)$ accurately.

\section{Conclusions}

This paper considered the relationship between a supplier and a customer operating under a service agreement. The supplier carries a certain amount of safety stock to fulfill the service agreement, whereas the customer is able to reduce its demand variability, albeit by incurring an extra cost. Since a reduction in the customer demand variability reduces the safety stock of the supplier, the supplier is willing to offer a price discount. We studied a pricing scheme where the supplier returns a fraction of its potential cost savings from a reduction in the customer demand variability back to the customer through a price discount. We showed that both the supplier and the customer benefit from such a pricing scheme and the cost savings of the supplier are larger than those of the customer. If the two parties repeatedly use our pricing scheme, then the customer eventually keeps the standard deviation of its demand at the level that minimizes the total system cost. Our pricing scheme continues to provide cost savings to both the supplier and the customer even when the customer does not reveal the cost component $\mathcal{C}(\cdot)$ accurately. Numerical illustrations showed that our pricing scheme is especially beneficial to the supplier and the customer when the uncertainty in the customer demand costs highly either to the supplier or to the customer, or when the cost of reducing the uncertainty in the customer demand is small. Furthermore, the customer can significantly increase its cost savings by not revealing the cost component $\mathcal{C}(\cdot)$ accurately.

\section{Appendix}

Proof of Lemma 1 We now show the third part. By (7), we have $\dot{\mathcal{C}}(X(-\dot{\mathcal{C}}(b)-h))-\dot{\mathcal{C}}(b)-h+h=0$, which implies that $X(-\dot{\mathcal{C}}(b)-h)=b$. Letting $\varepsilon>0$, if $r=-\dot{\mathcal{C}}(b)-h-\varepsilon$, then the derivative of 
the objective function of problem $(5)$ at $x=b$ is $\dot{\mathcal{C}}(b)-\dot{\mathcal{C}}(b)-h-\varepsilon+h<0$, which implies that $X(-\dot{\mathcal{C}}(b)-h-\varepsilon)=b$. Therefore, over the interval $(-\infty,-\dot{\mathcal{C}}(b)-h]$, the objective function of problem (6) becomes $g b-[\bar{p}-r \bar{x}+r b]$, which, noting that $\bar{x} \leq b$, is decreasing in $r$. Also, the right-hand derivative of the objective function of problem $(6)$ at $r=-\dot{\mathcal{C}}(b)-h$ is

$$
\begin{aligned}
g \dot{X}(-\dot{\mathcal{C}}(b)-h)+\bar{x}-[-\dot{\mathcal{C}}(b)-h] \dot{X}(-\dot{\mathcal{C}}(b)-h)-X(-\dot{\mathcal{C}}(b)-h) & \\
& =[\dot{\mathcal{C}}(b)+g+h] \dot{X}(-\dot{\mathcal{C}}(b)-h)+\bar{x}-b<0
\end{aligned}
$$

where we use the fact that $\dot{X}(\cdot)<0$ and the assumption that $\dot{\mathcal{C}}(b)+g+h>0$. Therefore, the optimal solution cannot lie in the interval $(-\infty,-\dot{\mathcal{C}}(b)-h]$. By using the same argument, one can show that the optimal solution cannot lie in the interval $[-\dot{\mathcal{C}}(0)-h, \infty)$ either.

Proof of Lemma 2 Since $0<x^{s}<\bar{x} \leq b$ and $\ddot{\mathcal{C}}(\cdot)>0$, (4) implies that $\dot{\mathcal{C}}(0)+h<\dot{\mathcal{C}}\left(x^{s}\right)+h=$ $-g<\dot{\mathcal{C}}(\bar{x})+h \leq \dot{\mathcal{C}}(b)+h$. Therefore, $-\dot{\mathcal{C}}(\bar{x})-h$ and $g$ both lie in the interval $[-\dot{\mathcal{C}}(b)-h,-\dot{\mathcal{C}}(0)-h]$, over which the objective function of problem (6) is differentiable. To show that $r_{o}>-\dot{\mathcal{C}}(\bar{x})-h$, we show that the derivative of the objective function of problem (6) at $-\dot{\mathcal{C}}(\bar{x})-h$ is strictly negative. By (7), we have $\dot{\mathcal{C}}(X(-\dot{\mathcal{C}}(\bar{x})-h))-\dot{\mathcal{C}}(\bar{x})-h+h=0$, which implies that $X(-\dot{\mathcal{C}}(\bar{x})-h)=\bar{x}$. Since $\ddot{\mathcal{C}}(\cdot)>0$, we have $\dot{\mathcal{C}}(\bar{x})+g+h>\dot{\mathcal{C}}\left(x^{s}\right)+g+h=0$. Thus, the derivative of the objective function of problem $(6)$ at $-\dot{\mathcal{C}}(\bar{x})-h$ is $g \dot{X}(-\dot{\mathcal{C}}(\bar{x})-h)+\bar{x}-[-\dot{\mathcal{C}}(\bar{x})-h] \dot{X}(-\dot{\mathcal{C}}(\bar{x})-h)-X(-\dot{\mathcal{C}}(\bar{x})-h)=$ $[\dot{\mathcal{C}}(\bar{x})+g+h] \dot{X}(-\dot{\mathcal{C}}(\bar{x})-h)<0$, where the last inequality uses the fact that $\dot{X}(\cdot)<0$. Similarly, to show that $r_{o}<g$, we show that the derivative of the objective function of problem (6) at $g$ is strictly positive. By (7), we have $\dot{\mathcal{C}}(X(g))+g+h=0=\dot{\mathcal{C}}\left(x^{s}\right)+g+h$, which implies that $X(g)=x^{s}$. Thus, the derivative of the objective function of problem (6) at $g$ is $g \dot{X}(g)+\bar{x}-g \dot{X}(g)-X(g)=\bar{x}-x^{s}>0$. We now show that $x^{s}<x_{o}<\bar{x}$. By (4), (7) and the fact that $r_{o}<g$, we have $\dot{\mathcal{C}}\left(x^{s}\right)+g+h=0=$ $\dot{\mathcal{C}}\left(X\left(r_{o}\right)\right)+r_{o}+h<\dot{\mathcal{C}}\left(X\left(r_{o}\right)\right)+g+h$. Since $\ddot{\mathcal{C}}(\cdot)>0$, this chain of inequalities yields $x^{s}<X\left(r_{o}\right)=x_{o}$. Similarly, by (7) and the fact that $r_{o}>-\dot{\mathcal{C}}(\bar{x})-h$, we have $\dot{\mathcal{C}}\left(X\left(r_{o}\right)\right)-\dot{\mathcal{C}}(\bar{x})<\dot{\mathcal{C}}\left(X\left(r_{o}\right)\right)+r_{o}+h=0$. Since $\ddot{\mathcal{C}}(\cdot)>0$, we obtain $x_{o}=X\left(r_{o}\right)<\bar{x}$. This establishes the first part.

The assumption that $\dot{\mathcal{C}}(0)+g+h<0$ and the first part imply that $\dot{\mathcal{C}}(0)<-g-h<-r_{o}-h<\dot{\mathcal{C}}(\bar{x})$. Therefore, there exists an $\widetilde{x} \in(0, \bar{x})$ such that $\dot{\mathcal{C}}(\widetilde{x})=-r_{o}-h$. Thus, since $\ddot{\mathcal{C}}(\cdot)>0$, we have $\mathcal{C}(\widetilde{x})-\left[r_{o}+h\right][\bar{x}-\widetilde{x}]=\mathcal{C}(\widetilde{x})+\dot{\mathcal{C}}(\widetilde{x})[\bar{x}-\widetilde{x}]<\mathcal{C}(\bar{x})$, which implies that $\mathcal{C}(\widetilde{x})+h \widetilde{x}+\bar{p}-r_{o} \bar{x}+r_{o} \widetilde{x}<$ $\mathcal{C}(\bar{x})+h \bar{x}+\bar{p}$.

Proof of Lemma 6 We now consider the cases $\dot{\mathcal{C}}(0)+\frac{r+h}{\lambda}>0$ and $\dot{\mathcal{C}}(b)+\frac{r+h}{\lambda}<0$. If $\dot{\mathcal{C}}(0)+r+h>0$, then the derivative of the objective function of problem (5) is strictly positive at $x=0$ and we have $X(r)=0$. Therefore, if $\dot{\mathcal{C}}(0)+\frac{r+h}{\lambda}-h+h>0$, then we have $X\left(\frac{r+h}{\lambda}-h\right)=0$. On the other hand, if $\dot{\mathcal{C}}(0)+\frac{r+h}{\lambda}>0$, then the derivative of the objective function of problem (12) is strictly positive at $x=0$ and the optimal solution to problem (12) is also 0 . Therefore, the optimal solution to problem 
(12) is $X\left(\frac{r+h}{\lambda}-h\right)$ when $\dot{\mathcal{C}}(0)+\frac{r+h}{\lambda}>0$. By using the same argument, one can also check that the optimal solution to problem (12) is $X\left(\frac{r+h}{\lambda}-h\right)$ when $\dot{\mathcal{C}}(b)+\frac{r+h}{\lambda}<0$.

Proof of Lemma 7 Both parts follow by Lemma 1, because if the customer reveals the cost component $\mathcal{C}(\cdot)$ as $\lambda \mathcal{C}(\cdot)$, then the supplier makes its computations assuming that the cost component $\mathcal{C}(\cdot)$ is $\lambda \mathcal{C}(\cdot)$. Thus, we can assume that the cost component $\mathcal{C}(\cdot)$ is $\lambda \mathcal{C}(\cdot)$ and apply Lemma 1.

Lemma 11 (This lemma is useful for proving Lemma 8.) Letting $M(\lambda)=[R(\lambda)+h] / \lambda$, we have $\dot{M}(\lambda)<0$ and $\dot{R}(\lambda)>0$ for all $\lambda>0$.

Proof of Lemma 11 Dropping the constant term, the objective function of problem (14) can be written as $[g+h] X\left(\frac{r+h}{\lambda}-h\right)+r \bar{x}-\lambda F\left(\frac{r+h}{\lambda}-h\right)$, where we let $F(r)=[r+h] X(r)$. Since $R(\lambda)$ is the optimal solution to problem (14), it satisfies the first order condition

$$
\frac{g+h}{\lambda} \dot{X}\left(\frac{R(\lambda)+h}{\lambda}-h\right)+\bar{x}-\dot{F}\left(\frac{R(\lambda)+h}{\lambda}-h\right)=0 .
$$

Differentiating the expression above with respect to $\lambda$, we obtain

$$
-\frac{g+h}{\lambda^{2}} \dot{X}\left(\frac{R(\lambda)+h}{\lambda}-h\right)+\dot{M}(\lambda)\left\{\frac{g+h}{\lambda} \ddot{X}\left(\frac{R(\lambda)+h}{\lambda}-h\right)-\ddot{F}\left(\frac{R(\lambda)+h}{\lambda}-h\right)\right\}=0 .
$$

Since $\dot{X}(\cdot)<0$, the first term above is strictly positive. Since $\ddot{X}(\cdot)>0$ and $[r+h] X(r)$ is shown to be a concave function of $r$ in the proof of Lemma 1, the term in the curly brackets is strictly positive. Therefore, we must have $\dot{M}(\lambda)<0$.

Letting $K(r, \lambda)=\frac{1}{\lambda} \dot{X}\left(\frac{r+h}{\lambda}-h\right)$, the first order condition in (22) can be written as

$$
[g+h] K(R(\lambda), \lambda)+\bar{x}-\dot{F}\left(\frac{R(\lambda)+h}{\lambda}-h\right)=0 .
$$

We have

$$
\begin{aligned}
\frac{\partial K(r, \lambda)}{\partial \lambda} & =-\frac{1}{\lambda^{2}} \dot{X}\left(\frac{r+h}{\lambda}-h\right)-\frac{r+h}{\lambda^{3}} \ddot{X}\left(\frac{r+h}{\lambda}-h\right) \\
& =-\frac{1}{\lambda^{2}} \dot{X}\left(\frac{r+h}{\lambda}-h\right)\left\{1-\frac{[r+h] \dot{X}\left(\frac{r+h}{\lambda}-h\right) \dddot{\mathcal{C}}\left(X\left(\frac{r+h}{\lambda}-h\right)\right)}{\lambda \ddot{\mathcal{C}}\left(X\left(\frac{r+h}{\lambda}-h\right)\right)}\right\} \\
& =-\frac{1}{\lambda^{2}} \dot{X}\left(\frac{r+h}{\lambda}-h\right)\left\{1-\frac{\dot{\mathcal{C}}\left(X\left(\frac{r+h}{\lambda}-h\right)\right) \dddot{\mathcal{C}}\left(X\left(\frac{r+h}{\lambda}-h\right)\right)}{\left[\ddot{\mathcal{C}}\left(X\left(\frac{r+h}{\lambda}-h\right)\right)\right]^{2}}\right\}<0,
\end{aligned}
$$

where the second equality uses (9) and the third equality uses (8) and (13). The inequality follows from the fact that $\dot{X}(\cdot)<0$ and the assumption that $[\ddot{\mathcal{C}}(x)]^{2}<\dot{\mathcal{C}}(x) \dddot{\mathcal{C}}(x)$ for all $x \in[0, b]$. Differentiating (23) with respect to $\lambda$, we obtain

$$
\begin{aligned}
\left\{\left.[g+h] \frac{\partial K(r, \lambda)}{\partial r}\right|_{(R(\lambda), \lambda)}-\frac{1}{\lambda} \ddot{F}\left(\frac{R(\lambda)+h}{\lambda}\right)\right\} & \dot{R}(\lambda) \\
& +\left.[g+h] \frac{\partial K(r, \lambda)}{\partial \lambda}\right|_{(R(\lambda), \lambda)}+\frac{R(\lambda)+h}{\lambda^{2}} \ddot{F}\left(\frac{R(\lambda)+h}{\lambda}-h\right)=0 .
\end{aligned}
$$


Since $\partial K(r, \lambda) / \partial r=\frac{1}{\lambda^{2}} \ddot{X}\left(\frac{r+h}{\lambda}-h\right)>0$ and $F(\cdot)$ is concave, the term in the curly brackets above is strictly positive. Among the last two terms, the first one is strictly negative due to (24). The second one is also negative, because $R(\lambda)+h>-\lambda \dot{\mathcal{C}}(b)>0$ by the second part of Lemma 7 and $F(\cdot)$ is concave. Therefore, we must have $\dot{R}(\lambda)>0$.

Proof of Lemma 8 To show that $1<\lambda_{o}<-[g+h] / \dot{\mathcal{C}}(\bar{x})$, we show that the derivative of the objective function of problem (15) is strictly negative over the interval $(0,1]$ and strictly positive over the interval $[-[g+h] / \dot{\mathcal{C}}(\bar{x}), \infty)$. We begin by noting that $R(-[g+h] / \dot{\mathcal{C}}(\bar{x}))=g$. To see this, noting $\dot{F}(r)=[r+h] \dot{X}(r)+X(r)$, we write the first order condition in (22) as

$$
\frac{g-R(\lambda)}{\lambda} \dot{X}\left(\frac{R(\lambda)+h}{\lambda}-h\right)=X\left(\frac{R(\lambda)+h}{\lambda}-h\right)-\bar{x}
$$

By (7), we have

$$
\dot{\mathcal{C}}\left(X\left(\frac{g+h}{-[g+h] / \dot{\mathcal{C}}(\bar{x})}-h\right)\right)-\dot{\mathcal{C}}(\bar{x})-h+h=0,
$$

which implies that $X\left(\frac{g+h}{-[g+h] / \dot{\mathcal{C}}(\bar{x})}-h\right)=\bar{x}$. Thus, $(25)$ is satisfied at $\lambda=-[g+h] / \dot{\mathcal{C}}(\bar{x})$ and $R(\lambda)=g$.

Letting $M(\lambda)=[R(\lambda)+h] / \lambda$, the derivative of the objective function of problem (15) is

$$
\begin{gathered}
\left\{\dot{\mathcal{C}}\left(X\left(\frac{R(\lambda)+h}{\lambda}-h\right)\right)+R(\lambda)+h\right\} \dot{X}\left(\frac{R(\lambda)+h}{\lambda}-h\right) \dot{M}(\lambda)+\dot{R}(\lambda)\left\{X\left(\frac{R(\lambda)+h}{\lambda}-h\right)-\bar{x}\right\} \\
=\left\{-\frac{R(\lambda)+h}{\lambda}+R(\lambda)+h\right\} \dot{X}\left(\frac{R(\lambda)+h}{\lambda}-h\right) \dot{M}(\lambda)+\frac{\dot{R}(\lambda)}{\lambda}[g-R(\lambda)] \dot{X}\left(\frac{R(\lambda)+h}{\lambda}-h\right) \\
=\dot{X}\left(\frac{R(\lambda)+h}{\lambda}-h\right)\left\{[R(\lambda)+h] \dot{M}(\lambda)\left[1-\frac{1}{\lambda}\right]+\frac{\dot{R}(\lambda)}{\lambda}[g-R(\lambda)]\right\}
\end{gathered}
$$

where the first equality uses (7) and (25). Examining some of the terms in (26) for $\lambda>0$, we have $\dot{X}\left(\frac{R(\lambda)+h}{\lambda}-h\right)<0$. By Lemma 7 , we have $R(\lambda)+h>-\lambda \dot{\mathcal{C}}(b)>0$. We also have $\dot{M}(\lambda)<0$ and $\dot{R}(\lambda)>0$ by Lemma 11. Finally, since $\bar{x}>x^{s}, \dot{\mathcal{C}}(\cdot)<0$ and $\ddot{\mathcal{C}}(\cdot)>0$, (4) implies that $-[g+h] / \dot{\mathcal{C}}(\bar{x})>-[g+h] / \dot{\mathcal{C}}\left(x^{s}\right)=1$.

Consider (26) for $\lambda \in(0,1]$. We have $1-1 / \lambda \leq 0$. Since $\dot{R}(\cdot)>0$, we also obtain $g-R(\lambda) \geq$ $g-R(1)>g-R(-[g+h] / \dot{\mathcal{C}}(\bar{x}))=0$. Therefore, $(26)$ is strictly negative for $\lambda \in(0,1]$. Similarly, consider (26) for $\lambda \in[-[g+h] / \dot{\mathcal{C}}(\bar{x}), \infty)$. We have $1-1 / \lambda>0$. Since $\dot{R}(\cdot)>0$, we also obtain $g-R(\lambda) \leq g-R(-[g+h] / \dot{\mathcal{C}}(\bar{x}))=0$. Therefore $(26)$ is strictly positive for $\lambda \in[-[g+h] / \dot{\mathcal{C}}(\bar{x}), \infty)$. This establishes that $1<\lambda_{o}<-[g+h] / \dot{\mathcal{C}}(\bar{x})$.

We have $-\lambda_{o} \dot{\mathcal{C}}(\bar{x})-h<r_{o}<g$ by Lemma 2, because if the customer reveals the cost component $\mathcal{C}(\cdot)$ as $\lambda_{o} \mathcal{C}(\cdot)$, then the supplier makes its computations assuming that the cost component $\mathcal{C}(\cdot)$ is $\lambda_{o} \mathcal{C}(\cdot)$. Thus, we can assume that the cost component $\mathcal{C}(\cdot)$ is $\lambda_{o} \mathcal{C}(\cdot)$ and apply Lemma 2 . 
Since $\lambda_{o}>1$ and $-\lambda_{o} \dot{\mathcal{C}}(\bar{x})-h<r_{o}<g$, we have

$$
-\dot{\mathcal{C}}(\bar{x})-h<\frac{r_{o}+h}{\lambda_{o}}-h<\frac{g+h}{\lambda_{o}}-h<g .
$$

Since $\dot{X}(\cdot)<0,(27)$ implies that $X(-\dot{\mathcal{C}}(\bar{x})-h)>X\left(\frac{r_{o}+h}{\lambda_{o}}-h\right)=x_{o}>X(g)$. By (4) and (7), we have $\dot{\mathcal{C}}(X(-\dot{\mathcal{C}}(\bar{x})-h))-\dot{\mathcal{C}}(\bar{x})-h+h=0$ and $\dot{\mathcal{C}}(X(g))+g+h=0=\dot{\mathcal{C}}\left(x^{s}\right)+g+h$, which imply that $X(-\dot{\mathcal{C}}(\bar{x})-h)=\bar{x}$ and $X(g)=x^{s}$. Therefore, we have $\bar{x}>x_{o}>x^{s}$. This establishes the first part.

Since $\dot{\mathcal{C}}(\cdot)<0$, the first part implies that $\lambda_{o} \dot{\mathcal{C}}(0)<\dot{\mathcal{C}}(0)<-g-h<-r_{o}-h<\lambda_{o} \dot{\mathcal{C}}(\bar{x})$. Therefore, there exists an $\widetilde{x} \in(0, \bar{x})$ such that $\lambda_{o} \dot{\mathcal{C}}(\widetilde{x})=-r_{o}-h$. Thus, since $\lambda_{o} \ddot{\mathcal{C}}(\cdot)>0$, we have $\lambda_{o} \mathcal{C}(\widetilde{x})-\left[r_{o}+h\right][\bar{x}-\widetilde{x}]=\lambda_{o} \mathcal{C}(\widetilde{x})+\lambda_{o} \dot{\mathcal{C}}(\widetilde{x})[\bar{x}-\widetilde{x}]<\lambda_{o} \mathcal{C}(\bar{x})$, which implies that $\lambda_{o} \mathcal{C}(\widetilde{x})+h \widetilde{x}+\bar{p}-$ $r_{o} \bar{x}+r_{o} \widetilde{x}<\lambda_{o} \mathcal{C}(\bar{x})+h \bar{x}+\bar{p}$.

Proof of Proposition 9 We first show the second part. Since $\lambda_{o} \ddot{\mathcal{C}}(\cdot)>0$ and $\lambda_{o} \mathcal{C}(\widetilde{x})+h \widetilde{x}+\bar{p}-$ $r_{o} \bar{x}+r_{o} \widetilde{x}<\lambda_{o} \mathcal{C}(\bar{x})+h \bar{x}+\bar{p}$, we have $\lambda_{o} \dot{\mathcal{C}}(\bar{x})[\widetilde{x}-\bar{x}]<\lambda_{o} \mathcal{C}(\widetilde{x})-\lambda_{o} \mathcal{C}(\bar{x})<-\left[r_{o}+h\right][\widetilde{x}-\bar{x}]$. Having $\lambda_{o} \dot{\mathcal{C}}(\bar{x})[\widetilde{x}-\bar{x}]<-\left[r_{o}+h\right][\widetilde{x}-\bar{x}]$ implies that $\widetilde{x}-\bar{x}<0$, because if we have $\widetilde{x}-\bar{x}>0$, then we obtain $\lambda_{o} \dot{\mathcal{C}}(\bar{x})<-\left[r_{o}+h\right]$ and this contradicts Lemma 8. Since $\lambda_{o}>1$ by Lemma $8, \dot{\mathcal{C}}(\cdot)<0$ and $\widetilde{x}-\bar{x}<0$, we have $0<\mathcal{C}(\widetilde{x})-\mathcal{C}(\bar{x})<\lambda_{o} \mathcal{C}(\widetilde{x})-\lambda_{o} \mathcal{C}(\bar{x})<-\left[r_{o}+h\right][\widetilde{x}-\bar{x}]$, which implies that $\mathcal{C}(\widetilde{x})+h \widetilde{x}+\bar{p}-r_{o} \bar{x}+r_{o} \widetilde{x}<\mathcal{C}(\bar{x})+h \bar{x}+\bar{p}$. Therefore, the customer is strictly better off under the standard deviation-price pair $\left(\widetilde{x}, \bar{p}-r_{o} \bar{x}+r_{o} \widetilde{x}\right)$. The fact that the supplier is strictly better off under this standard deviation-price pair and the first part can be shown by using the same argument used to show Proposition 3.

Lemma 12 (This lemma is useful for proving Proposition 10.) Assume that the sequences $\left\{x_{n}\right\}$ and $\left\{p_{n}\right\}$ are generated through $x_{n+1}=X\left(\frac{R_{n}\left(\lambda_{n}\right)+h}{\lambda_{n}}-h\right)$ and $p_{n+1}=p_{n}-R_{n}\left(\lambda_{n}\right) x_{n}+R_{n}\left(\lambda_{n}\right) x_{n+1}$, where $R_{n}\left(\lambda_{n}\right)$ and $\lambda_{n}$ are as defined in (17) and (18), and $x_{1}=\bar{x}, p_{1}=\bar{p}$. Let $M_{n}(\lambda)=\left[R_{n}(\lambda)+h\right] / \lambda$. Then, there exist $L_{1}, U_{1}, L_{2}, U_{2}, L_{3}, U_{3}, L_{4}, U_{4}, L_{5}$ and $U_{6}$ such that, for all $n$, we have

$$
\begin{gathered}
0<L_{1} \leq-\dot{\mathcal{C}}\left(x_{n}\right) \leq U_{1}<\infty \\
0<L_{2} \leq \ddot{\mathcal{C}}\left(x_{n}\right) \leq U_{2}<\infty \\
0<L_{3} \leq \lambda_{n} \leq U_{3}<\infty \\
0<L_{4} \leq R_{n}\left(\lambda_{n}\right)+h \leq U_{4}<\infty \\
0<L_{5} \leq-\dot{M}_{n}\left(\lambda_{n}\right) \\
\dot{R}_{n}\left(\lambda_{n}\right) \leq U_{6}<\infty .
\end{gathered}
$$

Proof of Lemma 12 By Lemma 8, we have $x^{s}<x_{2}=X\left(\frac{R_{1}\left(\lambda_{1}\right)+h}{\lambda_{1}}-h\right)<x_{1}$. Starting from the standard deviation-price pair $\left(x_{2}, p_{2}\right)$ and reapplying Lemma 8, we have $x^{s}<x_{3}=X\left(\frac{R_{2}\left(\lambda_{2}\right)+h}{\lambda_{2}}-\right.$ 
$h)<x_{2}$. Continuing in this fashion, we obtain $x^{s}<\ldots<x_{n+1}<x_{n}<\ldots<x_{1}$. Therefore, $x_{n} \in\left[x^{s}, x_{1}\right]$ for all $n$. Since $0<-\dot{\mathcal{C}}(x)<\infty$ and $0<\ddot{\mathcal{C}}(x)<\infty$ for all $x \in[0, b]$, we can set $L_{1}=\min _{x \in\left[x^{s}, x_{1}\right]}-\dot{\mathcal{C}}(x), U_{1}=\max _{x \in\left[x^{s}, x_{1}\right]}-\dot{\mathcal{C}}(x), L_{2}=\min _{x \in\left[x^{s}, x_{1}\right]} \ddot{\mathcal{C}}(x)$ and $U_{2}=\max _{x \in\left[x^{s}, x_{1}\right]} \ddot{\mathcal{C}}(x)$.

Since $x_{n}>x^{s}$ for all $n$, the first part of Lemma 8 implies that $1<\lambda_{n}<-[g+h] / \dot{\mathcal{C}}\left(x_{n}\right)$ for all $n$. Therefore, we can set $L_{3}=1$ and $U_{3}=[g+h] / L_{1}$. By $(7)$, we have

$$
\dot{\mathcal{C}}\left(X\left(\frac{R_{n}\left(\lambda_{n}\right)+h}{\lambda_{n}}-h\right)\right)+\frac{R_{n}\left(\lambda_{n}\right)+h}{\lambda_{n}}-h+h=\dot{\mathcal{C}}\left(x_{n+1}\right)+\frac{R_{n}\left(\lambda_{n}\right)+h}{\lambda_{n}}=0 .
$$

Therefore, we can set $L_{4}=L_{1} L_{3}$ and $U_{4}=U_{1} U_{3}$.

Since $0<-\dddot{\mathcal{C}}(x)<\infty$ for all $x \in[0, b]$, letting $L_{7}=\min _{x \in\left[x^{s}, x_{1}\right]}-\dddot{\mathcal{C}}(x)$ and $U_{7}=\max _{x \in\left[x^{s}, x_{1}\right]}-\dddot{\mathcal{C}}(x)$, we have $0<L_{7} \leq-\dddot{\mathcal{C}}\left(x_{n}\right) \leq U_{7}<\infty$ for all $n$. By (8), we have

$$
\dot{X}\left(\frac{R_{n}\left(\lambda_{n}\right)+h}{\lambda_{n}}-h\right) \ddot{\mathcal{C}}\left(x_{n+1}\right)+1=0 .
$$

Therefore, $1 / U_{2} \leq-\dot{X}\left(\frac{R_{n}\left(\lambda_{n}\right)+h}{\lambda_{n}}-h\right) \leq 1 / L_{2}$ for all $n$. By $(9)$, we have

$$
\ddot{X}\left(\frac{R_{n}\left(\lambda_{n}\right)+h}{\lambda_{n}}-h\right) \ddot{\mathcal{C}}\left(x_{n+1}\right)+\left[\dot{X}\left(\frac{R_{n}\left(\lambda_{n}\right)+h}{\lambda_{n}}-h\right)\right]^{2} \dddot{\mathcal{C}}\left(x_{n+1}\right)=0 .
$$

Therefore, $L_{7} / U_{2}^{3} \leq \ddot{X}\left(\frac{R_{n}\left(\lambda_{n}\right)+h}{\lambda_{n}}-h\right) \leq U_{7} / L_{2}^{3}$ for all $n$.

Letting $F(r)=[r+h] X(r)$, we have

$$
\ddot{F}\left(\frac{R_{n}\left(\lambda_{n}\right)+h}{\lambda_{n}}-h\right)=2 \dot{X}\left(\frac{R_{n}\left(\lambda_{n}\right)+h}{\lambda_{n}}-h\right)+\frac{R_{n}\left(\lambda_{n}\right)+h}{\lambda_{n}} \ddot{X}\left(\frac{R_{n}\left(\lambda_{n}\right)+h}{\lambda_{n}}-h\right) .
$$

The proof of Lemma 1 shows that $\ddot{F}(\cdot) \leq 0$. Therefore,

$$
0 \leq-\ddot{F}\left(\frac{R_{n}\left(\lambda_{n}\right)+h}{\lambda_{n}}-h\right) \leq \frac{2}{L_{2}}-\frac{L_{4} L_{7}}{U_{3} U_{2}^{3}} .
$$

We set $U_{8}=2 L_{2}^{-1}-L_{4} L_{7} U_{3}^{-1} U_{2}^{-3}$.

Similar to (22), since $R_{n}(\lambda)$ is the optimal solution to problem (17), it satisfies

$$
\frac{g+h}{\lambda} \dot{X}\left(\frac{R_{n}(\lambda)+h}{\lambda}-h\right)+x_{n}-\dot{F}\left(\frac{R_{n}(\lambda)+h}{\lambda}-h\right)=0 .
$$

Differentiating the expression above with respect to $\lambda$ and evaluating the derivative at $\lambda=\lambda_{n}$, we obtain

$$
-\frac{g+h}{\lambda_{n}^{2}} \dot{X}\left(\frac{R_{n}\left(\lambda_{n}\right)+h}{\lambda_{n}}-h\right)+\dot{M}_{n}\left(\lambda_{n}\right)\left[\frac{g+h}{\lambda_{n}} \ddot{X}\left(\frac{R_{n}\left(\lambda_{n}\right)+h}{\lambda_{n}}-h\right)-\ddot{F}\left(\frac{R_{n}\left(\lambda_{n}\right)+h}{\lambda_{n}}-h\right)\right]=0 .
$$

Therefore, we can set

$$
L_{5}=\frac{\frac{[g+h]}{U_{3}^{2} U_{2}}}{\frac{[g+h] U_{7}}{L_{3} L_{2}^{3}}+U_{8}} .
$$


Let $K(r, \lambda)=\frac{1}{\lambda} \dot{X}\left(\frac{r+h}{\lambda}-h\right)$. By $(24)$, we have

$0<-\left.\frac{\partial K(r, \lambda)}{\partial \lambda}\right|_{\left(R_{n}\left(\lambda_{n}\right), \lambda_{n}\right)}=-\frac{1}{\lambda_{n}^{2}} \dot{X}\left(\frac{R_{n}\left(\lambda_{n}\right)+h}{\lambda_{n}}-h\right)\left\{\frac{\dot{\mathcal{C}}\left(x_{n+1}\right) \dddot{\mathcal{C}}\left(x_{n+1}\right)}{\left[\ddot{\mathcal{C}}\left(x_{n+1}\right)\right]^{2}}-1\right\} \leq \frac{1}{L_{3}^{2} L_{2}}\left\{\frac{U_{1} U_{7}}{L_{2}^{2}}-1\right\}$.

We also have

$$
\left.\frac{\partial K(r, \lambda)}{\partial r}\right|_{\left(R_{n}\left(\lambda_{n}\right), \lambda_{n}\right)}=\frac{1}{\lambda_{n}^{2}} \ddot{X}\left(\frac{R_{n}\left(\lambda_{n}\right)+h}{\lambda_{n}}-h\right) \geq \frac{L_{7}}{U_{3}^{2} U_{2}^{3}} .
$$

We set $U_{9}=L_{3}^{-2} L_{2}^{-1}\left[U_{1} U_{7} L_{2}^{-2}-1\right]$ and $L_{10}=L_{7} U_{3}^{-2} U_{2}^{-3}$.

Similar to (23), since $R_{n}(\lambda)$ is the optimal solution to problem (17), it satisfies

$$
[g+h] K\left(R_{n}(\lambda), \lambda\right)+x_{n}-\dot{F}\left(\frac{R_{n}(\lambda)+h}{\lambda}-h\right)=0 .
$$

Differentiating the expression above with respect to $\lambda$ and evaluating the derivative at $\lambda=\lambda_{n}$, we obtain

$$
\begin{aligned}
\left\{\left.[g+h] \frac{\partial K(r, \lambda)}{\partial r}\right|_{\left(R_{n}\left(\lambda_{n}\right), \lambda_{n}\right)}\right. & \left.-\frac{1}{\lambda_{n}} \ddot{F}\left(\frac{R_{n}\left(\lambda_{n}\right)+h}{\lambda_{n}}\right)\right\} \dot{R}_{n}\left(\lambda_{n}\right) \\
& +\left.[g+h] \frac{\partial K(r, \lambda)}{\partial \lambda}\right|_{\left(R_{n}\left(\lambda_{n}\right), \lambda_{n}\right)}+\frac{R_{n}\left(\lambda_{n}\right)+h}{\lambda_{n}^{2}} \ddot{F}\left(\frac{R_{n}\left(\lambda_{n}\right)+h}{\lambda_{n}}-h\right)=0 .
\end{aligned}
$$

Therefore, we can set

$$
U_{6}=\frac{[g+h] U_{9}+\frac{U_{4}}{L_{3}^{2}} U_{8}}{[g+h] L_{10}}
$$

Proof of Proposition 10 As shown in the proof of Lemma 12, we have $x^{s}<\ldots<x_{n+1}<x_{n}<$ $\ldots<x_{1}$. Therefore, $\left\{x_{n}\right\}$ converges to some $x^{*} \geq x^{s}$. We want to show that $x^{*}=x^{s}$. Assume that $x^{*}>x^{s}$. Since $\ddot{\mathcal{C}}(\cdot)>0$ and $x^{s}<x^{*}<x_{n+1}$, (4) implies that $\dot{\mathcal{C}}\left(x_{n+1}\right)+g+h>\dot{\mathcal{C}}\left(x^{*}\right)+g+h>$ $\dot{\mathcal{C}}\left(x^{s}\right)+g+h=0$. We now show that this chain of inequalities implies that $x_{n}-x_{n+1}>U$ for all $n$ for some $U>0$, which contradicts the fact that $\left\{x_{n}\right\}$ converges.

Similar to (25), since $R_{n}(\lambda)$ is the optimal solution to problem (17), it satisfies

$$
\begin{aligned}
\frac{g-R_{n}\left(\lambda_{n}\right)}{\lambda_{n}} \dot{X}\left(\frac{R_{n}\left(\lambda_{n}\right)+h}{\lambda_{n}}-h\right)+x_{n} & -X\left(\frac{R_{n}\left(\lambda_{n}\right)+h}{\lambda_{n}}-h\right) \\
& =-\frac{g-R_{n}\left(\lambda_{n}\right)}{\lambda_{n} \ddot{\mathcal{C}}\left(X\left(\frac{R_{n}\left(\lambda_{n}\right)+h}{\lambda_{n}}-h\right)\right)}+x_{n}-X\left(\frac{R_{n}\left(\lambda_{n}\right)+h}{\lambda_{n}}-h\right) \\
& =-\frac{\lambda_{n} \dot{\mathcal{C}}\left(x_{n+1}\right)+g+h}{\lambda_{n} \ddot{\mathcal{C}}\left(x_{n+1}\right)}+x_{n}-x_{n+1}=0,
\end{aligned}
$$

where the first equality follows from (8) and the second equality follows from (28). Since $\ddot{\mathcal{C}}(\cdot)>0$ and $x_{n+1}>x^{*},(29)$ implies that

$$
\begin{aligned}
\lambda_{n} \ddot{\mathcal{C}}\left(x_{n+1}\right)\left[x_{n}-x_{n+1}\right]=\dot{\mathcal{C}}\left(x_{n+1}\right)+g+h+\left[\lambda_{n}-1\right] \dot{\mathcal{C}}\left(x_{n+1}\right) & \\
& >\dot{\mathcal{C}}\left(x^{*}\right)+g+h+\left[\lambda_{n}-1\right] \dot{\mathcal{C}}\left(x_{n+1}\right) .
\end{aligned}
$$


Since $x_{n}>x^{s}$, Lemma 8 implies that $\lambda_{n}>1$. If $\lambda_{n} \leq 1+\frac{\dot{\mathcal{C}}\left(x^{*}\right)+g+h}{2 U_{1}}$, where $U_{1}$ is as defined in Lemma 12, then we have

$$
\dot{\mathcal{C}}\left(x^{*}\right)+g+h+\left[\lambda_{n}-1\right] \dot{\mathcal{C}}\left(x_{n+1}\right) \geq \dot{\mathcal{C}}\left(x^{*}\right)+g+h-\frac{\dot{\mathcal{C}}\left(x^{*}\right)+g+h}{2 U_{1}} U_{1}=\frac{\dot{\mathcal{C}}\left(x^{*}\right)+g+h}{2}
$$

The expression above and (30) imply that

$$
x_{n}-x_{n+1}>\frac{\dot{\mathcal{C}}\left(x^{*}\right)+g+h}{2 \lambda_{n} \ddot{\mathcal{C}}\left(x_{n+1}\right)} \geq \frac{\dot{\mathcal{C}}\left(x^{*}\right)+g+h}{2 U_{3} U_{2}},
$$

where $U_{2}$ and $U_{3}$ are as defined in Lemma 12.

Let $M_{n}(\lambda)=\left[R_{n}(\lambda)+h\right] / \lambda$. Since $\lambda_{n}$ is the optimal solution to problem (18), it satisfies the first order condition

$$
\begin{aligned}
\left\{\dot{\mathcal{C}}\left(X\left(\frac{R_{n}\left(\lambda_{n}\right)+h}{\lambda_{n}}-h\right)\right)+R_{n}\left(\lambda_{n}\right)+h\right\} \dot{X}\left(\frac{R_{n}\left(\lambda_{n}\right)+h}{\lambda_{n}}-h\right) \dot{M}_{n}\left(\lambda_{n}\right) & \\
& +\dot{R}_{n}\left(\lambda_{n}\right)\left\{X\left(\frac{R_{n}\left(\lambda_{n}\right)+h}{\lambda_{n}}-h\right)-x_{n}\right\}=0
\end{aligned}
$$

which, by using the same argument used in (26), can be written as

$$
\dot{X}\left(\frac{R_{n}\left(\lambda_{n}\right)+h}{\lambda_{n}}-h\right)\left\{\left[R_{n}\left(\lambda_{n}\right)+h\right] \dot{M}_{n}\left(\lambda_{n}\right)\left[1-\frac{1}{\lambda_{n}}\right]+\frac{\dot{R}_{n}\left(\lambda_{n}\right)}{\lambda_{n}}\left[g-R_{n}\left(\lambda_{n}\right)\right]\right\}=0 .
$$

Since $\dot{X}(\cdot)<0,(32)$ implies that $g-R_{n}\left(\lambda_{n}\right)=-\left\{\left[R_{n}\left(\lambda_{n}\right)+h\right] \dot{M}_{n}\left(\lambda_{n}\right)\left[\lambda_{n}-1\right]\right\} / \dot{R}_{n}\left(\lambda_{n}\right)$. Therefore, noting (28) and (30), we have

$$
\lambda_{n} \ddot{\mathcal{C}}\left(x_{n+1}\right)\left[x_{n}-x_{n+1}\right]=\lambda_{n} \dot{\mathcal{C}}\left(x_{n+1}\right)+g+h=g-R_{n}\left(\lambda_{n}\right)=-\frac{\left[R_{n}\left(\lambda_{n}\right)+h\right] \dot{M}_{n}\left(\lambda_{n}\right)\left[\lambda_{n}-1\right]}{\dot{R}_{n}\left(\lambda_{n}\right)} .
$$

If $\lambda_{n}>1+\frac{\dot{\mathcal{C}}\left(x^{*}\right)+g+h}{2 U_{1}}$, the equality above implies that

$$
x_{n}-x_{n+1}>\frac{L_{4} L_{5} \frac{\dot{\mathcal{C}}\left(x^{*}\right)+g+h}{2 U_{1}}}{U_{3} U_{2} U_{6}}
$$

where $L_{4}, L_{5}$ and $U_{6}$ are as defined in Lemma 12. Letting

$$
U=\left[\dot{\mathcal{C}}\left(x^{*}\right)+g+h\right] \min \left\{\frac{1}{2 U_{3} U_{2}}, \frac{L_{4} L_{5}}{2 U_{1} U_{3} U_{2} U_{6}}\right\}>0
$$

(31) and (33) imply that $x_{n}-x_{n+1}>U$, which contradicts the fact that $\left\{x_{n}\right\}$ converges. Therefore, we must have $x^{*}=x^{s}$.

By Lemma 8, we have $1<\lambda_{n}<-[g+h] / \dot{\mathcal{C}}\left(x_{n}\right)$ for all $n$. By (4) and the fact that $\lim _{n \rightarrow \infty} x_{n}=x^{s}$, we have $\lim _{n \rightarrow \infty} \dot{\mathcal{C}}\left(x_{n}\right)=\dot{\mathcal{C}}\left(x^{s}\right)=-[g+h]$. Thus, we obtain $\lim _{n \rightarrow \infty} \lambda_{n}=1$. 


\section{References}

A. Balakrishnan, J. Geunes, and M. S. Pangburn. Coordinating supply chains by controlling upstream variability propogation. Manufacturing $\mathscr{E}$ Service Operations Management, 6(2):163-183, 2004.

J. W. M. Bertrand. Balancing production level variations and inventory variations in complex production systems. International Journal of Production Research, 24(5):1059-1074, 1986.

K. E. Bourland, S. G. Powell, and D. F. Pyke. Exploiting timely demand information to reduce inventories. European Journal of Operational Research, 92:239-253, 1999.

R. N. Boute, S. M. Disney, M. R. Lambrecht, and B. van Houdt. An integrated production and inventory model to dampen upstream demand variability in supply chain. European Journal of Operational Research, 178:121-142, 2007.

G. Cachon. Competitive supply chain inventory management. In S. Tayur, R. Ganeshan, and M. Magazine, editors, Quantitative Methods for Supply Chain Management, Boston, 1998. Kluwer.

G. P. Cachon and M. L. Fisher. Supply chain inventory management and the value of shared information. Management Science, 46(8):1032-1048, 2000.

F. Chen. Market segmentation, advanced demand information, and supply chain performance. Manufacturing $\&$ Service Operations Management, 3(1):53-67, 2001.

K. L. Cheung. A continuous review inventory model with a time discount. IIE Transactions, 30: 747-757, 1998 .

M. Dada and K. N. Srikanth. Pricing policies for quantity discounts. Management Science, 33(10): $1247-1252,1987$.

J. Dejonckheere, S. M. Disney, M. R. Lambrecht, and D. R. Towill. Measuring and avoiding the bullwhip effect: A control theoretic approach. European Journal of Operational Research, 147: 567-590, 2003.

S. M. Disney, I. Farasyn, M. Lambrecht, D. R. Towill, and W. Van de Velde. Taming the bullwhip effect whilst watching customer service in a single supply chain echelon. European Journal of Operational Research, 173:151-172, 2006.

S. Gavirneni, R. Kapuscinski, and S. Tayur. Value of information in capacitated supply chains. Management Science, 45(1):16-24, 1999.

S. M. Gilbert and R. H. Ballou. Supply chain benefits from advanced customer commitments. Journal of Operations Management, 18:61-73, 1999.

S. C. Graves, D. B. Kletter, and W. B. Hetzel. A dynamic model for requirements planning with application to supply chain optimization. Operations Research, 46S(3):S35-S49, 1998.

R. Gullu. On the value of information in dynamic production/inventory problems under forecast evolution. Naval Research Logistics, 43:289-303, 1996.

R. Gullu. A two-echelon allocation model and the value of information under correlated forecasts and demands. European Journal of Operational Research, 99:386-400, 1997.

R. Hariharan and P. Zipkin. Customer order information, lead times and inventories. Management Science, 41:1599-1607, 1995.

F. Karaesmen, J. A. Buzacott, and Y. Dallery. Integrating advance order information in make-tostock production systems. IIE Transactions, 34:649-662, 2002.

F. Karaesmen, G. Liberopoulos, and Y. Dallery. The value of advance demand information in production/inventory systems. Annals of Operations Research, 126:135-157, 2004.

T. D. Klastorin, K. Moinzadeh, and J. Son. Coordinating orders in supply chains through price discounts. IIE Transactions, 34:679-689, 2002.

R. Lal and R. Staelin. An approach for developing an optimal discount pricing policy. Management Science, 30(12):1524-1539, 1984.

H. L. Lee and M. J. Rosenblatt. A generalized quantity discount pricing model to increase supplier's profits. Management Science, 32(9):1177-1185, 1986. 
H. L. Lee, K. C. Soo, and C. S. Tang. The value of information sharing in a two-level supply chain. Management Science, 46(5):626-643, 2000.

J. P. Monahan. A quantity discount pricing model to increase vendor profits. Management Science, 30(6):720-726, 1984.

O. Ozer. Replenishment strategies for distribution systems under advance demand information. Management Science, 49(3):255-272, 2003.

U. W. Thonemann. Improving supply-chain performance by sharing advance demand information. European Journal of Operational Research, 142:81-107, 2002.

A. Tsay, S. Nahmias, and N. Agrawal. Modeling supply chain contracts: A review. In S. Tayur, R. Ganeshan, and M. Magazine, editors, Quantitative Methods for Supply Chain Management, Boston, 1998. Kluwer.

K. Van Donselaar, L. R. Kopczak, and M. Wouters. The use of advance demand information in a project-based supply chain. European Journal of Operational Research, 130:519-538, 2001.

Z. K. Weng. Channel coordination and quantity discounts. Management Science, 41(9):1509-1522, 1995.

X. Zhang. The impact of forecasting methods on the bullwhip effect. International Journal of Production Economics, 88:15-27, 2004.

P. Zipkin. Foundations of Inventory Management. McGraw-Hill, Boston, MA, 2000. 